\title{
BELIEF CONTRACTION \\ IN THE CONTEXT OF THE GENERAL THEORY OF RATIONAL CHOICE
}

\author{
HANS ROTT
}

\begin{abstract}
This paper reorganizes and further develops the theory of partial meet contraction which was introduced in a classic paper by Alchourron. Gärdenfors, and Makinson. Our purpose is threefold. First. we put the theory in a broader perspective by decomposing it into two layers which can respectively be treated by the general theory of choice and preference and elementary model theory. Second. we reprove the two main representation theorems of AGM and present two more representation results for the finite case that "lie between" the former. thereby partially answering an open question of AGM. Our method of proof is uniform insofar as it uses only one form of "revealed preference". and it explains where and why the finiteness assumption is needed. Third. as an application, we explore the logic characterizing theory contractions in the finite case which are governed by the structure of simple and prioritized belief bases.
\end{abstract}

$\S 1$. Introduction. The theory of partial meet contraction and revision was developed by Alchourrón, Gärdenfors, and Makinson (henceforth, "AGM") in a paper [1] published in this journal in 1985. That paper is the by now classic reference of the flourishing research programme of theory change, or as it is alternatively called, belief revision [4], [5], [6], [11]. In particular, it has been shown that partial meet contraction is a powerful tool for the reconstruction of other kinds of theory change such as safe contraction, epistemic entrenchment contraction, and base contraction [2], [18]-[21].

The basic idea of partial meet contraction is as follows. In order to eliminate a proposition $x$ from a theory $A$ while obeying the constraint of deductive closure and minimizing the loss of information, it is plausible to look at the maximal subsets $B$ of $A$ that fail to imply x. In an earlier paper, Alchourrón and Makinson had proved that when $A=C n(A)$ taking one such $B$ leaves an agent with too many propositions, while taking the intersection of all such $B$ 's leaves him with too few. In [1], AGM investigate the idea of taking the intersection of a select set of such B's. The choice of which B's to take is made with the help of a selection function. A natural question is whether all these selections can be represented as the selections of preferred $B$ 's, where the preferences between maximally nonimplying subsets of $A$ are independent of the proposition $x$ to be deleted.

Received June 1. 1992; revised October 29, 1992.

Key words and phrases. Theory change, belief revision. rational choice, preference, partial meet contraction, base contraction. $0022-4812935804-0020503.50$ 
The purpose of the present paper is threefold. First, we put the theory of partial meet contraction in a broader perspective. We decompose it into two layers, each of which can be cultivated with the help of methods developed in different research areas. On the one hand, in relating selection functions to preference relations we can draw on existing work in social choice theory [3], [10], [23], [24]. On the other hand, we shail elaborate on a remark of Grove [8] and link maximally nonimplying subsets to "possible worlds" or models, thereby making it possible to compare partial meet contraction with semantical approaches to belief revision and nonmonotonic reasoning [11]-[13], [16]. Exaggerating somewhat, we can say that the theory of partial meet contraction emerges from juxtaposing the general theory of rational choice $(\$ 2)$ and a tiny bit of model theory $(\$ 3)$. After introducing abstract postulates for contraction furctions in $\$ 4$, we reprove the iwo main representation theorems of $[1, \mathrm{pp} .521,530]$ concerning partial meet contraction and transitively relational partial meet contraction in a slightly more systematic fashion $(\$ 5)$.

Second, we provide a partial solution to a problem left unanswered by AGM and still considered to be an interesting open question in [17, p. 195]. More precisely, we present two new results that lie strictly "between" those of [1], viz., representation theorems for relational and negatively transitiveiy relational partial meet contraction (still in \$5). However, these results hold only under certain preconditions. If the theory to be contracted is logically finite, then all these conditions are met. Our decomposition allows for a uniform method of proof using so-called Samuelson preferences. It increases our understanding of the partial meet contraction mechanism by localizing exactly in which parts of the proofs the finiteness assumption is effective.

Third, as an application, we explore the logic of a variant of syntax-based belief change, namely, simple and prioritized base contractions in the finite case (\$6). The basic idea here is that real life theories are generated from finite axiomatizations and that the axioms may carry different epistemic weight. Both the syntactical encoding of a theory and the prioritization are held to be relevant for theory change. We achieve a logical characterization of simple and prioritized base contraction by combining one of our representation theorems with observations originating with Lewis [15] and Nebel [18], [19].

Independently from the research for this article, related work has been carried out by Katsuno and Mendelson [11] and Lindström [16]. Lindström proves numerous representation theorems, and like us, he heavily draws on results from the general theory of rational choice. However, there are some important differences. He adopts a semantic approach right from the outset, while our reconstruction starts by turning a syntactic approach into an essentially semantic one, with the notable feature that any two "worlds" in our sense are linguistically distinguishable (our models are "injective", to use a term of Michael Freund). Lindström's main concern is nonmonotonic inference relations and the related area of belief revision, whereas we shall focus on belief contraction. (Everything we might wisis to say about the intimate connection between revisions and contractions is contained in [1] and [5].) He applies liberal maximization where we apply stringent maximization. He uses different postulates for choice functions and a different notion of 
revealed preference. The main contrast between his approach and ours. however, is that Lindström permits revisions by - possibly infinite - sets of propositions whilst we stick to the original AGM model of changing theories by single propositions. Lindström's generalization allows him to revise by "worlds" and thus dispense with a finiteness assumption which will prove to be necessary at several places in the AGM framework. In fact, it is a major aim of our paper to make transparent the reasons where and why logical finiteness is needed in the AGM theory. Further important results on purely finitistic belief revision are found in [11] by Katsuno and Mendelson, who incorporate a revision operator into their object language. Both papers are highly recommended and should be read in conjunction with the present one.

Unfortunately, space limitations do not allow a presentation that makes for easy reading. Familiarity with earlier work either in the area of theory changean excellent overview is [5] - or in the general theory of rational choice-of particular relevance is [10] - will greatly facilitate the reader's task. However, we will shortly repeat the basic definitions so that our presentation is in principle selfcontained. It may be useful to inform the reader in advance about the fundamental entities that will show up as determinants of theory change. We shall meet contraction functions - , selection functions $\gamma$, preference relations $\leq$ (and $\preceq \prec$ ), maximally nonimplying sets $M$ (and $N$ ), maximally consistent sets or "worlds" $W$, as well as simple and prioritized belief bases $B$ and $\left\langle B_{i}\right\rangle$. We shall frequently jump to and fro between these kinds of entities. When we wish to generate a contraction function, selection function, preference relation, maximal nonimplying set, world, or belief $b$ ase from another kind of entity, we shall use metalevel mappings denoted by $\mathscr{C}, \mathscr{S}, \mathscr{P}, \mathscr{M}, \mathscr{W}, \mathscr{B}$, respectively.

\section{§. Selection functions and preference relations.}

2.1. General selection functions. Let $X$ be a set and $X$ be a nonempty subset of $2^{X}-\{\varnothing\}$. A selection function (or choice function) over $\mathscr{X}$ is a function $\gamma: \mathscr{X} \rightarrow 2^{X}$ such that $\gamma(S)$ is a nonempty subset of $S$, for every $S \in \mathscr{X}$. Intuitively, selection functions are supposed to give us the best elements of each $S$ in $\mathscr{X}$. The requirement that $\gamma(S)$ be nonempty means that a selection function is effective or "diecisive": In every case it reaches a decision of which elements are best. As Lindström [16] points out, the decisiveness of selection functions corresponds to a condition of consistency preservation in belief revision and nonmonotonic reasoning. Here consistency is judged by some monotonic background consequence relation, not necessarily by the classical one (cf. subsection 3.1).

Domain conditions for selection functions are important. A set $\mathscr{X}$ of subsets of $X$ is called $n$-covering $(n=1,2,3, \ldots)$ if it contains all subsets of $X$ with exactly $n$ elements, $\mathscr{X}$ is called $n_{1} n_{2}$-covering (or $n_{1} n_{2} n_{3}$-covering) if it is $n_{1}$-covering and $n_{2}$-covering (and $n_{3}$-covering). $\mathscr{X}$ is called $\omega$-covering if it is $n$-covering for all natural numbers $n=1,2,3, \ldots$. The set $\mathscr{X}$ is called additive if it is closed under arbitrary unions, and it is called finitely additive if it is closed under finite unions; it is called subtractive if for every $S$ and $S^{\prime}$ in $\mathscr{X}$ such that $S \nsubseteq S^{\prime}, S-S^{\prime}$ is also in $\mathscr{X}$, 
and it is called full if $x=2^{X}-\{\varnothing\}$. Of course, if $x$ is 1 -covering and finitely additive, then it is $\omega$-covering. Finally, we say that $x$ is compact if for every $T$ and $S_{i}, i \in I$, from $x$, if $T \subseteq \bigcup\left\{S_{i}: i \in I\right\}$ then $T \subseteq \bigcup\left\{S_{i}: i \in I_{0}\right\}$ for some finite $I_{0} \subseteq I$. We say that a selection function $;$ with domain $x$ is $n$-covering, additive, subtractive, etc., if its domain $\mathscr{X}$ is $n$-covering, additive, subtractive, etc.

2.2. Constraints on selection functions. We consider the following "coherence postulates" for selection functions. Their motivation, where it is not obvious, is given in [10], [23], [24].

(I) For all $S, S^{\prime} \in \mathscr{X}$, if $S \subseteq S^{\prime}$, then $S \cap \gamma_{i}\left(S^{\prime}\right) \subseteq \gamma_{i}(S)$.

("Chernoff's axiom", Sen's Property $x$ )

(II) For all $\left\{S_{i}: i \in I\right\} \subseteq X$ such that $\left.\bigcup_{\{} S_{i}: i \in I\right\} \in \mathscr{X}, \bigcap\left\{;\left(S_{i}\right): i \in I\right\} \subseteq$ $i\left(\bigcup\left\{S_{i}: i \in I\right\}\right)$.

(III) For all $S, S^{\prime} \in \mathscr{X}$ such that $S \subseteq S^{\prime}$, if $i^{\prime}\left(S^{\prime}\right) \subseteq i(S)$, then $\gamma(S) \subseteq \gamma^{\prime}\left(S^{\prime}\right)$.

(Sen`s Property $\gamma)$

("Superset Axion", Sen's Property $\varepsilon$ )

(IV) For all $S, S^{\prime} \in \mathscr{X}$ such that $S \subseteq S^{\prime}$, if $\gamma^{\prime}\left(S^{\prime}\right) \cap S \neq \varnothing$, then $\gamma(S) \subseteq \gamma\left(S^{\prime}\right)$.

("Arrow's axiom", Sen's Property $\beta+$ )

Condition (III) is independent of condition (II), even for finite $X$ and in the presence of condition (I). For example, let $X=\{x, y, z\}, X$ be the power set of $X$ minus $\varnothing$, and consider $i_{1}$ and $\gamma_{2}$ defined by $i_{1}(\{x, y\})=i_{2}(\{x, y\})=\{x, y\}$, $\gamma_{1}(\{x, z\})=\{x\}, \gamma_{1}(\{y, z\})=\{z\}, \gamma_{1}(\{x, y, z\})=\{x\}, \gamma_{2}(\{x, z\})=\{x, z\}, \gamma_{2}(\{y, z\})=$ $\{y, z\}$, and $i_{2}(\{, x, y, z\})=\{x, y\}$. Evidently, $i_{1}$ satisfies (I) and (II) and violates (III), while $i_{2}$ satisfies (I) and (III) and violates (II). On the other hand, condition (IV) implies condition (II): let $\left.\left\{S_{i}: i \in I\right\} \subseteq \mathscr{X}, \bigcup_{\{} S_{i}: i \in I\right\} \in \mathscr{X}$. Now $\varnothing \neq i\left(\left(\bigcup\left\{S_{i}: i \in I\right\}\right)\right.$ $\subseteq \bigcup\left\{S_{i}: i \in I\right\}$, so $i\left(\bigcup\left\{S_{i}: i \in I\right\}\right) \cap S_{i_{0}} \neq \varnothing$ for some $i_{0} \in I$. So by (IV), $\left.\bigcap_{\{i}\left(S_{i}\right): i \in I\right\} \subseteq i\left(S_{i_{0}}\right) \subseteq i\left(\left(\bigcup_{i}\left\{S_{i}: i \in I\right\}\right)\right.$. It is trivial to see that condition (IV) also implies condition (III), since $\varnothing \neq i_{i}\left(S^{\prime}\right) \subseteq i^{\prime}(S)$ implies $i^{\prime}\left(S^{\prime}\right) \cap S \neq \varnothing$.

2.3. Rational choice. In the general theory of choice and preference we often find an idea which can be phrased in the slogan "Rational choice is relational choice". That is, rational choice is choice which can be construed as based on an underlying preference relation.

The intended interpretation of the set $i(S)$, called the choice set for $S$, is that its elements are regarded as equally adequate or satisfactory choices for an agent whose values are represented by the function $i$, and who faces a decision problem represented by the set $S$. Following Chernoff [3]..., this relativistic concept of equiadequacy for a given decision problem bears sharp distinction from invariant concepts like preferential matching or indifference which for a given agent are not relativized to decision problems, and which may be subject to more stringent constraints, both for rational agents and for agents in general ([10, p. 189], notation adapted).

Choice sets are taken to be sets of "best" elements. There are basically two ideas to make this precise. The first is based on a nonstrict (reflexive) preference relation $\leq$ :

$$
\eta(S)=\left\{y \in S: y^{\prime} \leq y \text { for all } y^{\prime} \in S\right\} .
$$


The second idea is based on a strict (asymmetric) preference relation $<$ :

$$
\gamma(S)=\left\{y \in S: y \nless y^{\prime} \text { for all } y^{\prime} \in S\right\} .
$$

These suggestions are respectively referred to as stringent and liberal maximization in [10, p. 197] and G-rationality and M-rationality in [24, p. 21]. (Stringent maximization is often attributed to Condorcet, 1785.) If $<$ is the converse complemen $^{i}$ of $\leq$, then stringent and liberal maximization coincide. If $<$ is the asymmetric part of $\leq$, then every liberal maximizer with respect to $<$ is a stringent maximizer with respect to the augmentation $\leq^{+}$of $\leq$which is defined by $x \leq^{+} y$ iff $x \leq y$ or $y \leq x$, i.e., iff not $y<x$. Clearly, $\leq^{+}$is connected, i.e., it holds for every $x$ and $y$ that either $x \leq^{+} y$ or $y \leq^{+} x$. While $\leq$ allows us to keep apart indifferences (both $x \leq y$ and $y \leq x$ ) from incomparabilities (neither $x \leq y$ nor $y \leq x$ ), $\leq^{+}$ blurs just this distinction, for whenever we have neither $x \leq y$ nor $y \leq x$ we have both $x \leq^{+} y$ and $y \leq^{+} x$. Thus, we may not expect $\leq^{+}$to be transitive. If, on the other hand, $\leq$ is already connected then $\leq^{+}=\leq$, and stringent maximization witil respect to the asymmetric part $<$ of $\leq$ coincides with liberal maximization with respect to $\leq$. For all this, cf. $[10, \$ 3]$.

In accordance with [1] as well as with the dominant approach in the theory of choice and preference, we shall focus on stringent maximization. ${ }^{1}$ From now on, when we say that $\gamma$ is relational with respect to $\leq$ over $X$, we mean that $\gamma(S)=$ $\left\{y \in S: y^{\prime} \leq y\right.$ for all $\left.y^{\prime} \in S\right\}$ for every $S \in \mathscr{X}$. In this case we write $\gamma=\mathscr{Y}(\leq)$. When we say that $\gamma$ is relational, we mean that there is some relation $\leq$ over $X$ such that $\gamma=\mathscr{P}(\leq)$.

For any nonstrict relation $\leq,<$ is used to denote the asymmetric part of $\leq$, which is defined by $x<y$ iff $x \leq y$ and not $y \leq x$. $\leq$ is called $n$-acyclic, if no $n$ objects $x_{1}, x_{2}, \ldots, x_{n}$ form a cycle under $<$, i.e., if a chain $x_{1}<x_{2}<\cdots<x_{::}<x_{1}$ does not occur. 1-Acyclicity is irreflexivity, 2-acyclicity is asymmetry. $\leq$ is called acyclic, if it is $n$-acyclic for every $n=1,2,3, \ldots \leq$ is called negatively acyclic if there is no cycle under $\not$, i.e., if never $x_{1} \not \leq x_{2} \not \leq \cdots \not x_{n} \not \leq x_{1}$, and it is called negatively well-founded if there is no infinite descending chain under $\not$, i.e., if never $\cdots \not x_{3} \not \leq x_{2} \not \leq x_{1}$. For connected relations $\leq$, negative well-foundedness coincides with converse well-foundedness where infinite ascending chains $x_{1}<x_{2}<$ $x_{3}<\cdots$ do not occur. Obviously, if $\leq$ is conversely (or negatively) well-founded, then it is acyclic (negatively acyclic). $\leq$ is smooth with respect to $X$ if there are no infinite descending $\not$-chains in $S$, for every $S \in \mathscr{X}$. Smoothness is a restricted form of negative well-foundedness. $\leq$ is called negaticely transitice (virtually connected, modular, ranked) if $x \not \leq y$ and $y \leq z$ implies $x \not z z$. It is quickly verified that a con-

\footnotetext{
${ }^{1}$ Intuitively, however, I think that liberal maximization is preferable. Liberal maximization is based on strict relations which do not allow to distinguish between incomparabilities and indifferences. Nonstrict relations do make this distinction, but stringent maximization tends to require connected relations which often can be had only if incomparabilities are turned into indifferences--i.e.. if augmentations are used. The interpretation of nonstrict relations as the converse complements of - more intuitive strict relations explains the crucial role of negative transitivity and negative well-foundedness in the following. Also compare the recommendation in [22] to regard the nonstrict epistemic entrenchment relation $\leq_{E}$ of $[7]$ as the converse complement of a more intuitive strict relation $<_{E}$.
} 
nected relation $\leq$ is negatively transitive iff it is quasi-transitive in the sense that its asymmetric part $<$ is transitive. Quasi-transitively is a much disputed requirement in social choice theory [10], [23], [24]. It should be noted that all transitive relations $\leq$ are both acyclic and quasi-transitive.

2.4. Two kinds of revealed preferences. In many contexts one can hope to recover the underlying preferences of an agent from observed choice behavior. The two most commonly used types of "revealed preference" relations are the Samuelson preferences (Samuelson 1950)

$$
\begin{aligned}
\leq_{i} & =\left\{\left\langle x, x^{\prime}\right\rangle \in X \times X: \exists S \in \mathscr{X} \text { such that }\left\{x, x^{\prime}\right\} \subseteq S \text { and } x^{\prime} \in i(S)\right\} \\
& =\bigcup\{S \times \gamma(S): S \in \mathscr{X}\}
\end{aligned}
$$

and the so-called base preferences (Uzawa 1956, Arrow 1959):

$$
\begin{aligned}
\leq_{i, 2} & =\left\{\left\langle x, x^{\prime}\right\rangle \in X \times X:\left\{x, x^{\prime}\right\} \in X \text { and } x^{\prime} \in i_{i}\left(\left\{x, x^{\prime}\right\}\right)\right\} \\
& =\bigcup\{S \times \gamma(S): S \in X \text { and } S \text { has at most two elements }\} .
\end{aligned}
$$

The terminology is taken from [10], where many other possibilities of defining notions of revealed preference are discussed. Obviously, $x \leq_{i, 2} y$ implies $x \leq_{i ;} y$. Notice that neither of these relations is guaranteed to be reflexive, unless $\gamma$ is 1 covering. $\leq_{i, 2}$ is defined for arbitrary $\gamma$ 's, but the definition makes good sense only for 2 -covering ones for which $\left\{x, x^{\prime}\right\}$ is always in $X$. In this case, base preferences and Samuelson preferences are connected. Given a selection function $\gamma$, we shall also denote $\leq_{\gamma}$ by $\mathscr{P}(\gamma)$ and $\leq_{i .2}$ by $\mathscr{P}_{2}(\gamma){ }^{2}$

2.5. Some basic properties and reformulations. The following lemmas list a number of important facts which are basically common knowledge in the general theory of rational choice (cf. [10], [23], [24]). The straightforward proofs are omitted (but can be obtained from the author).

LEMMA 1. (a) If $i$ is relational, then it satisfies (I) and (II).

(b) If $\gamma$ is 12-covering and satisfies (I) and (II), then $\gamma=\mathscr{H}\left(\mathscr{P}_{2}(\gamma)\right)$.

(c) If $\gamma$ is 12-covering and satisfies (I), then $\mathscr{P}(\gamma)=\mathscr{P}_{2}(\gamma)$.

(d) If $\gamma$ is 12-covering and satisfies (I) and (II), then $\gamma=\mathscr{P}(\mathcal{P}(\gamma))$.

(e) If $\gamma$ is 12-covering and relational, then $\gamma=\mathscr{H}(: \mathcal{P}(\gamma))=\mathscr{H}(\mathscr{P} 2(\gamma))$.

(f) If $\gamma$ is additive and satisfies (I) and (II), then $\gamma=\mathscr{P}(\mathscr{P}(\gamma))$.

Lemma 2. If $\gamma$ is $12 n$-covering and satisfies $(\mathrm{I})$, then $\mathscr{P}(\gamma)=\mathscr{P}_{2}(\gamma)$ is $n$-acyclic. If $\gamma$ is $\omega$-covering and satisfies (I), then $\mathscr{P}(\gamma)$ is acyclic.

(b) If $\gamma$ is 123-covering and satisfies (I) and (III), then $\mathscr{P}(\gamma)$ is negatively transitive.

(c) If $\gamma$ is finitely additive and satisfies (IV), then $\mathscr{P}(\gamma)$ is transitive.

${ }^{2}$ It is worth pointing out that the characteristic definition of a relation of epistemic entrenchment (see [7], [22]) between propositions from an observed contraction behavior, viz.

$$
x \leq_{E} y \quad \text { iff } \quad x \notin A-(x \wedge y) \text { or } y \in A-(x \wedge y)
$$

can also be interpreted as a base preference [22, p. 61]. In that paper it is argued that the instruction "remove $x \wedge y$ " should be regarded as an instruction to remove $x$ or remove $y$, where the agent holding theory $A$ has free choice which proposition(s) out of $\{x, y\}$ to remove. 
LEMMA 3. (a) If $\leq$ is smooth with respect to $\mathscr{X}$, then $\mathscr{S}(\leq)$ is a selection function over $\mathscr{X}$ which satisfies (I) and (II).

(b) If $\leq$ is negatively transitive and negatively well-founded (or if $\leq$ is negatively transitive and smooth and $\mathscr{X}$ is subtractive), then $\mathscr{S}(\leq)$ satisfies (III).

(c) If $\leq$ is transitive, then $\mathscr{S}(\leq)$ satisfies (IV).

Now we bring conditions (I)-(IV) into a form which is more suitable for our purposes.

$\left(\mathrm{I}^{\prime}\right)$ For all $S, S^{\prime} \in \mathscr{X}$ such that $S \cup S^{\prime} \in \mathscr{X}, \gamma\left(S \cup S^{\prime}\right) \subseteq \gamma(S) \cup \gamma\left(S^{\prime}\right)$.

(II') For all $S, S^{\prime} \in \mathscr{X}$ such that $S \cup S^{\prime} \in \mathscr{X}, \gamma^{\prime}(S) \cap \gamma^{\prime}\left(S^{\prime}\right) \subseteq \gamma\left(S \cup S^{\prime}\right)$.

(III') For all $S \in \mathscr{X}$ and $S^{\prime}$ such that $S \cup S^{\prime} \in \mathscr{X}$, if $\gamma\left(S \cup S^{\prime}\right) \cap S^{\prime}=\varnothing$, then $\gamma(S) \subseteq \gamma\left(S \cup S^{\prime}\right)$.

$\left(I V^{\prime}\right)$ For all $S \in \mathscr{X}$ and $S^{\prime}$ such that $S \cup S^{\prime} \in \mathscr{X}$, if $\gamma\left(S \cup S^{\prime}\right) \cap S \neq \varnothing$, then $\gamma(S) \subseteq \gamma\left(S \cup S^{\prime}\right)$.

(I\&II) For all $S, S_{i} \in \mathscr{X}, i \in I$, if $S \subseteq \bigcup\left\{S_{i}: i \in I\right\}$, then $S \cap \bigcap\left\{\gamma\left(S_{i}\right): i \in I\right\} \subseteq \gamma(S)$.

(I\&II') For all $S, S^{\prime}, S^{\prime \prime} \in \mathscr{X}$, if $S \subseteq S^{\prime} \cup S^{\prime \prime}$, then $S \cap \gamma\left(S^{\prime}\right) \cap \gamma\left(S^{\prime \prime}\right) \subseteq \gamma(S)$.

Note that condition (II') is just a restriction of (II) to index sets with at most two elements, and similarly for (I\&II) and (I\&II').

LEMMA 4. (a) If $\gamma$ is subtractive, then condition (I) is equivalent to $\left(\mathrm{I}^{\prime}\right)$.

(b) If $\gamma$ is finitely additive, compact, and satisfies (I) and $\left(\mathrm{II}^{\prime}\right)$, then $\gamma=\mathscr{P}(\mathscr{P}(\gamma))$.

(c) If $\gamma$ satisfies (I), then (III) is equivalent to (III').

(d) (IV) is equivalent to (IV').

(e) If $\gamma$ is additive, then the conjunction of (I) and (II) is equivalent to (I\&II).

(f) If $\gamma$ is finitely additive, then the conjunction of (I) and (II') is equivalent to $\left(\mathrm{I} \& \mathrm{II}^{\prime}\right)$.

Lemma $4(\mathrm{~b})$ is a substitute for Lemma $1(\mathrm{f})$ in the absence of infinite additivity. The conditions (I\&II) and (I\&II') are generalized forms of the conditions $(\gamma 7: \infty)$ and $(\gamma 7: 2)$ in [1]. AGM [1, Observation 4.10] noticed that (I\&II') is the key to relationality in partial meet contraction (because the domain $\mathscr{X}$ encountered there is compact), but as they failed to decompose that condition into the simpler ones, (I) and ( $\left.\mathrm{II}^{\prime}\right)$, it remained undigestible for them. As we shall see, they provided a postulate for contraction functions which corresponds to (I), but nothing in [1] corresponds to (II').

The results of the theory of rational choice are quite nice, and we may be optimistic about their applicability to theory change as determined by partial meet contractions. Still there are at least two problems which should not be underestimated. First, we do not know very much about selection functions which are neither 123covering nor additive. ${ }^{3}$ And second, in choice-and-preference theory we are faced with a bewildering pluralism of revealed preference relations (see [10]). It is hard to decide in advance which notion of revealed preference is "the" right notion for a given purpose. It will turn out, happily, that the relation $\mathscr{P}(\gamma)$ defined above and, to a smaller degree, the relation $\mathscr{P}_{2}(\gamma)$ which is equivalent in the finite case (in the sense of subsection 3.2) are suitable for partial meet contractions.

\footnotetext{
${ }^{3}$ This is no problem for Lindström [16] whose selection functions are always $\omega$-covering. Consequently, Lindström's constructions can always make use of the base preferences $\mathscr{P}_{2}(\gamma)$.
} 


\section{§3. Selection functions and preference relations in partial meet contraction.}

3.1. The general case. We presuppose a propositional language $L$, with the usual connectives $\neg, \wedge, \vee, \rightarrow$ and $\leftrightarrow$. From now on, small roman letters $a, b, c, \ldots, x, y, z, \ldots$ denote propositions of $L$, and capital roman letters $A, B, A^{\prime}, B^{\prime}, \ldots$, $M, N, M^{\prime}, N^{\prime} \ldots, W, W^{\prime} \ldots$ denote sets of propositions. Notational conventions: Capital $A$ is used to denote a theory, $B$ an arbitrary set of propositions (in general, a nontheory), $M$ and $N$ maximally nonimplying subsets (of some $A$ or $B$, respectively), and $W$ a maximally consistent set of $L$. All these metavariables may occur with primes or subscripts.

As in [1], $L$ is assumed to be governed by some reflexive, monotonic and idempotent logic (consequence operation) $C n$ which is supraclassical and compact and satisfies the deduction theorem: $B \subseteq C n(B)$; if $B \subseteq B^{\prime}$, then $C n(B) \subseteq C n\left(B^{\prime}\right)$; $C n(C n(B))=C n(B)$; if $C n_{0}$ is classical tautological implication, then $C n_{0}(B) \subseteq C n(B)$; if $y \in C n(B)$, then $y \in C n\left(B_{0}\right)$ for some finite subset $B_{0}$ of $B$; and finally, $y \in$ $C n\left(B \cup\left\{x_{j}^{\prime}\right)\right.$ iff $x \rightarrow y \in C n(B)$. That is, $C n$ is a classical logic in the sense of [16]. Note that in the present context, idempotence is equivalent to the cut rule, and the deduction theorem is equivalent to disjunction in the antecedents.

We also write $B \vdash x$ for $x \in C n(B)$. $A$ is a called a theory if $A=C n(A)$. By $\llbracket x \rrbracket$ and $[B]$ we denote the set of all "possible worlds" (valuations, models) in which $x$ (every $x$ in $B$ ) is true. Here and throughout this paper, we identify a "world" satisfying $x$ or $B$ with a maximally $C n$-consistent set of propositions which contains $x$ (respectively, $B$ ). The set of all "worlds" is denoted $\mathbf{W}$.

Let $B \perp x=\{M \subseteq B: x \notin C n(M)$ and $x \in C n(N)$ for all $N$ with $M \subset N \subseteq B\}$. Note that the elements of $B \perp x$ are theories if $B$ is a theory. If $\forall x$, then $B \perp x$ is nonempty, by the compactness of $C n^{4}$ We define $B \perp=\{B \perp x: x \in C n(B)-$ $C n(\varnothing)\}$ and $U_{B}=\bigcup(B \perp)=\bigcup\{B \perp x: x \in C n(B)-C n(\varnothing)\}$. (Here we slightly deviate from the definitions in [1] which also include $x$ 's from $C n(\varnothing)$.) If $C n(B) \neq$ $C n(\varnothing)$, then $B \perp$ is a nonempty subset of $2^{2^{13}}-\{\varnothing\}$. The case $C n(B)=C n(\varnothing)$ will be handled separately in our reconstruction of partial meet contraction.

Lemma 5. Let $A$ he a theory and $x \in A$. Then $M \in A \perp x$ iff there is a maximally consistent set ("world") $W$ such that $\neg x \in W$ and $M=A \cap W$.

For the proof, compare $\$ 4$ of Grove [8].

Now let $A$ again be a theory. For $M \in U_{A}$, put $\mathscr{V}^{\prime}(M)=C n(M \cup\{\neg x\})$, where $x$ is any proposition in $A-M$, and put . $\|(W)=W \cap A$, for any maximally consistent set $W$ such that $A \nsubseteq W$. The reader is invited to verify that $\mathscr{W}$ is well-defined(!), $\mathscr{W}$ is a bijection from $U_{A}$ to $V_{A}=\{W \in \mathbf{W}: A \nsubseteq W\}$, and.$/ l$ is the converse of $\mathscr{W}$, i.e., $\mathscr{U}(\mathscr{W}(M))=M$ and $\mathscr{W}(\mathscr{U}(W))=W$.

Given this representation of the elements of $U_{A}$, it is clear that they satisfy the following fullness and primeness conditions: If $x \in A-M$ and $y \in A$ then $x \rightarrow y \in M$,

${ }^{4}$ This marks a difference with Lewis [15] who identifies propositions with sets of extra-linguistic possible worlds and logical consequence with set-theoretic inclusion. Lacking compactness. Lewis has to ponder the impact of a "Limit Assumption" for premise semantics. 
and if $x, y \in A-M$, then $x \vee y \in A-M$ (cf. [1, Observations 6.1 and 6.2]). $U_{A}$ is just the set of all maximal proper subtheories of the theory $A$. Moreover, we immediately get

Corollary 1. Let $A$ be a theory and $x, y, y_{i}$ be in $A-C n(\varnothing)$.

(i) For $M \in U_{A}, x \notin M$ iff $M \in A \perp x$.

(ii) $A \perp(x \wedge y)=A \perp x \cup A \perp y$.

(iii) $A \perp(x \vee y)=A \perp x \cap A \perp y$.

(iv) $A \perp(x \vee \neg y)=A \perp x-A \perp y$.

(v) If $A \perp x \subseteq \bigcup\left\{A \perp y_{i}: i \in I\right\}$, then $A \perp x \subseteq \bigcup\left\{A \perp y_{i}: i \in I_{0}\right\}$ for some finite $I_{0} \subseteq I$.

Facts (i) and (ii) are contained in [1, Lemma 2.4 and Lemma 4.1]. We see that $A \perp$, the special domain $\mathscr{X}$ of the selection functions which will figure in partial meet contractions, is closed under finite unions, finite intersections, and differences. We give a direct proof of the compactness property (v). Let $A \perp x \subseteq \bigcup\left\{A \perp y_{i}: i \in I_{\}}\right.$. Then $\left\{y_{i}: i \in I\right\} \vdash x$. For otherwise, by Lindenbaum's Lemma and Lemma 5 , there would be an $M \in A \perp x$ such that $\left\{y_{i}: i \in I\right\} \subseteq M$, so $M \notin A \perp y_{i}$ for every $i$, contradicting our hypothesis. Compactness of $C n$ gives us $\left\{y_{i}: i \in I_{0}\right\} \vdash x$ for some finite $I_{0} \subseteq I$. Thus, there is, for every $M \in A \perp x$, an $i \in I_{0}$ such that $y_{i} \notin M$. Hence, by (i), there is, for every $M \in A \perp x$, an $i \in I_{0}$ such that $M \in A \perp y_{i}$. Thus, $A \perp x \subseteq$ $\bigcup\left\{A \perp y_{i}: i \in I_{0}\right\}$, as desired.

Now we can introduce selection functions for belief revision. Let $A$ be a theory. A selection function $\because: A \perp \rightarrow 2^{2^{A}}$ is called a selection function for $A$. It follows from Corollary 1 that every selection function $;$ for $A$ is finitely additive, subtractive, and compact.

Let $\gamma$ be a selection function for $A$. The completion $\gamma^{*}$ of $\gamma$ is defined by $\gamma^{*}(A \perp x)=\{M \in A \perp x: \bigcap \gamma(A \perp x) \subseteq M\}$ for all $x \in A-C n(\varnothing)$. Following [1], we call a selection function $;$ complete if $\gamma=i^{*}$. If $;$ is complete, then $M \in \gamma(A \perp x)$ whenever $\bigcap \gamma(A \perp x) \subseteq M \in U_{A}$. (Proof. Since $\{x \rightarrow y: y \in A\} \subseteq \bigcap \gamma(A \perp x)$, $\bigcap \gamma(A \perp x) \subseteq M$ implies $M \forall x$, so $M \in A \perp x$ by Corollary 1(i).)

A contraction function over a theory $A$ is a function $\dot{-}_{A}: L \rightarrow 2^{L}$. We write $A-x$ for $\dot{ }_{A}(x)$, and as there will be no danger of confusion, we shall often write just for $\dot{ }_{A}$. The term ' $A-x$ ' should be read as the 'the result of rationally removing $x$ from $A$ '. The idea of a contraction function dictates that it should satisfy at least the postulates $A-x \subseteq A$ and $x \notin A-x$ (unless $\vdash x$ ). More postulates will be discussed in $\S 4$. Intuitively, $A-x$ is the minimal, most economical or rational, change of $A$ needed to discard $x$.

A function - is the partial meet contraction function over A determined by the selection function $;$ for $A$ if and only if

$$
A-x= \begin{cases}\bigcap_{i}(A \perp x) & \text { for every } x \in A-C n(\varnothing), \\ A & \text { for every } x \notin A \text { and every } x \in C n(\varnothing) .\end{cases}
$$

Given a selection function $;$ for $A$, the partial meet contraction it determines will be denoted by $\mathscr{C}(i)$. There is a slight deviation from [1] in order to avoid the application of $;$ to $\varnothing$ and to preserve the perfect correspondence between $U_{A}$ and $V_{A}$. The function - is called a partial meet contraction oter $A$ if there is a selection function $;$ for $A$ such that $\dot{-}=6(;)$. 
A selection function ; for $A$ is called (transitively, quasi-transitively, connectively, acyclicly) relational over $A$ if there is a (transitive, quasi-transitive, connected, acyclic) preference relation $\leq$ over $U_{A}$ (over $2^{A}$ in [1]) such that for every $x \in A-C n(\varnothing)$,

$$
\gamma(A \perp x)=\left\{M \in A \perp x: M^{\prime} \leq M \text { for all } M^{\prime} \in A \perp x\right\} .
$$

This is an application of stringent maximization as discussed in subsection 2.3, and it is called the marking off identity in [1]. Recall that we write $;=\mathscr{P}(\leq)$ if $\gamma$ is determined by $\leq$ in that way.

A contraction function - is (transitively, quasi-transitively, connectively, acyclicly) relational if there is a (transitive, quasi-transitive, connected, acyclic) preference relation $\leq$ over $U_{A}$ such that $\dot{-}=\mathscr{6}(\mathscr{P}(\leq))$.

Using the above functions $\mathscr{W}^{\prime}$ and. $/ l$, we find that the selection $\gamma$ of sets in $A \perp x$ can equivalently be viewed as a selection $\gamma_{w}$ of maximally consistent sets, or "worlds", in $[\neg x]$. If we define

$$
i_{w}(\mathbb{[} \neg x \rrbracket)=\{\mathscr{W}(M): M \in \gamma(A \perp x)\},
$$

then clearly,

$$
\ddot{\gamma}(A \perp x)=\left\{\mathscr{l}(W): W \in \gamma_{W}(\llbracket \neg x \rrbracket)\right\}
$$

In the principal case $x \in A-C n(\varnothing), A-x=A \cap \bigcap\left(\gamma_{w}([\neg x])\right)$. Conditions for the partial meet mechanism may thus be viewed as model theoretic or semantical conditions. The "possible worlds" view facilitates a visualization of the conditions on preference relations and selection functions and relates the work on partial meet contraction directly to the minimal models approach as adopted in $[11]-[16]$.

A set $\mathbf{W}^{\prime}$ of maximal consistent sets of propositions is called elementary ( $\Delta$ elementary) if there is a proposition $y$ (a set of propositions $B$ ) such that $\mathbf{W}^{\prime}$ is exactly the set of all maximally consistent sets of propositions which contain $y$ (respectively, which include $B)$, in symbols $\mathbf{W}^{\prime}=\llbracket y \rrbracket\left(\mathbf{W}^{\prime}=\llbracket B \rrbracket\right)$. We call $\gamma$ elementary $\left(\Delta\right.$-elementary) if $\left.\gamma_{W}(\llbracket \neg x \rrbracket)\right)$ is elementary $(\Delta$-elementary) for every $x \in A-C n(\varnothing)$. If $\gamma$ is elementary $\left(\Delta\right.$-elementary) and $\gamma_{W}(\llbracket \neg x \rrbracket)=\llbracket y \rrbracket\left(\gamma_{W}(\llbracket \neg x \rrbracket)=\llbracket B \rrbracket\right)$, then $A-x=C n(\{z \vee y: z \in A\})=A \cap C n(y)$ (respectively, $A-x=C n\left(\left\{z \vee z^{\prime}: z \in A\right.\right.$ and $\left.\left.z^{\prime} \in B_{j}^{\prime}\right)=A \cap C n(B)\right)$.

REMARK 1. $\gamma$ is complete iff it is $\Delta$-elementary.

Proof. From left to right. We show that $\llbracket(\bigcap \hat{\gamma}(A \perp x)) \cup\{\neg x\} \rrbracket=\gamma_{W}(\llbracket \neg x \rrbracket)$, for every $x \in A-C n(\varnothing)$. Clearly, $(\bigcap \gamma(A \perp x)) \cup\{\neg x\} \subseteq W$ for every $W \in \gamma_{W}(\llbracket \neg x \rrbracket)$. To show the converse, suppose for reductio that $(\bigcap \gamma(A \perp x)) \cup$ $\{\neg x\} \subseteq W$ and $W \notin \gamma_{W}(\llbracket \neg x \rrbracket)$. Hence, by the latter, $\mathscr{M}(W) \notin \gamma(A \perp x)$, so by the completeness of $\gamma, \bigcap \gamma(A \perp x) \nsubseteq \mathscr{U}(W)$. But also $(\bigcap \gamma(A \perp x)) \cup\{\neg x\} \subseteq W$, and $\bigcap \gamma(A \perp x) \subseteq A$, so $\bigcap \gamma(A \perp x) \subseteq \mathscr{U}(W)$, and we have a contradiction.

From right to left. Suppose for reductio that there are $x \in A-C n(\varnothing)$ and $M \in U_{A}$ such that $\bigcap \gamma(A \perp x) \subseteq M$ and $M \notin \gamma(A \perp x)$. Hence, $\mathscr{W}(M) \notin \gamma_{W}(\llbracket \neg x \rrbracket)$. Since $\gamma$ is $\Delta$-elementary, there is a set $B$ of propositions such that $\gamma_{W}(\llbracket \neg x \rrbracket)=\llbracket B \rrbracket$. 
So $B \nsubseteq \mathscr{W}(M)$. Take some $y \in B-\mathscr{W}(M)$. Since $x \vee y \notin \mathscr{W}^{\prime}(M)$, we get, by the definition of $\mathscr{W}, x \vee y \notin M$. So by $\bigcap \gamma(A \perp x) \subseteq M, x \vee y \notin \bigcap \gamma(A \perp x)$. But since $x \in A$ and $y \in \bigcap \gamma_{w}(\llbracket \neg x \rrbracket)$, we get $x \vee y \in A \cap \bigcap \gamma_{w}(\llbracket \neg x \rrbracket)=\bigcap \gamma(A \perp x)$, and we have again a contraciction.

As the domain $A \perp$ of selection functions for $A$ is finitely additive, subtractive, and compact, all domain requirements mentioned in Lemma 4(a)-(d) are satisfied. We can further adapt the conditions $\left(\mathrm{I}^{\prime}\right)-\left(\mathrm{IV}^{\prime}\right)$ to our needs. We now know that for all $x, y \in A-C n(\varnothing), A \perp x \cup A \perp y=A \perp(x \wedge y)$, and $\gamma(A \perp x) \cap A \perp y=\varnothing$ iff $y \in \bigcap \gamma(A \perp x)$. With $\mathscr{X}=A \perp$, the general conditions (I')-(IV') can therefore be transformed into the following conditions on selection functions for theories:

(I") For all $x, y \in A-C n(\varnothing), \gamma(A \perp(x \wedge y)) \subseteq \gamma(A \perp x) \cup \gamma(A \perp y)$.

(II") For all $x, y \in A-C n(\varnothing), \gamma(A \perp x) \cap \gamma(A \perp y) \subseteq \gamma(A \perp(x \wedge y))$.

(III") For all $x, y \in A-C n(\varnothing)$, if $y \in \bigcap \gamma(A \perp(x \wedge y))$, then $\gamma(A \perp x) \subseteq$ $\gamma(A \perp(x \wedge y))$.

$\left(I^{\prime \prime}\right)$ For all $x, y \in A-C n(\varnothing)$, if $x \notin \bigcap \gamma(A \perp(x \wedge y))$, then $\gamma(A \perp x) \subseteq$ $\gamma(A \perp(x \wedge y))$.

From these conditions, it will be a rather short step to some interesting postulates for rational ("economical") theory contraction.

3.2. The finite case. A set $B$ of propositions will be called logically finite (or finite modulo $C n$, or simply finite) if $C n$ partitions $B$ into finitely many cells. The finite case is much easier to handle than the general one. This is due to the fact that every selection function over a logically finite theory is $\omega$-covering and even full.

Notice that for a theory $A$ to be finite modulo a common logic $C n$, it will be necessary that the underlying language has only finitely many atoms. For suppose there are infinitely many atoms $p_{1}, p_{2}, p_{3}, \ldots$ in our language. Then for every proposition $x$ from $A$ there are infinitely many atoms $p_{i}$ not occurring in $x$. Thus the infinitely many $\left(x \vee p_{i}\right)$ 's which are all contained in $A$ will be mutually nonequivalent, so $A$ is not finite. Conversely, if only a finite number of nonequivalent propositional operators of bounded arity is definable in $C n$, as in classical propositional logic or in modal logics with finitely many modalities, then the finiteness of the number of propositional atoms is also sufficient for the logical finiteness of theories $A$ phrased in the language in question.

Given a logically finite theory $A$, it is clear that each of the following sets is finite: $A \perp x$, for every $x, A \perp$, and $U_{A}$.

A representative of a logically finite set of propositions $B$ is a conjunction of representatives of all the equivalence classes under $C n$ of propositions in $B$.

Henceforth, we shall use the following notational convention. For sets of propositions $A, B, \ldots, M, N, \ldots, W, \ldots$ which are finite modulo $C n$, the lower case letters $a, b, \ldots, m, n, \ldots, w, \ldots$ denote their representatives. For any two sets $A$ and $B$ such that $B \subseteq A, \bar{b}_{A}$ denotes the disjunction of representatives of those equivalence classes under $C n$, the elements of which are in $C n(A)-C n(B)$. When we are dealing with a fixed theory $A$, we simply write $\bar{b}$ instead of $\bar{b}_{A}$. If $B=A$, then $\bar{b}_{A}$ is defined to be the falsity, $\perp$. We may call $\bar{b}_{A}$ the corepresentative of $B$ (relative to $A$ ).

It is easy to verify in the finite case that for $M \in U_{A}$ and $W=\mathscr{W}(M), w$ is equivalent with $\neg a \wedge m$ and that for $W \in V_{A}$ and $M=\mathscr{U}(W), m$ is equivalent with $a \vee w$. This helps us to get the following useful 
Lemma 6. Let $A$ he a finite theory and $M \in U_{A}$.

(i) If 11 is the representatice of $y(M)$ and $\bar{w}_{L}$ is the corepresentative of $\|(M)$ relatice to the set of all propositions in L, then the following four propositions are equivalent: $\bar{m}_{A}, m \rightarrow a, \bar{w}_{L}$. and $\neg w$.

(ii) $A \perp \bar{m}=\{M\}$.

(iii) For all $M_{1} \ldots, M_{n} \in U_{A}$, if $m \vdash m_{1} \vee \cdots \vee m_{n}$, then $M=M_{i}$ for some $i$.

Lemma 6(ii), together with Corollary l(ii), shows that in the finite case every subset $\mathbf{M}$ of $U_{A}$ equals $A \perp x$ for some proposition $x$. viz., for $\left.x=\bigwedge i \bar{m}: M \in \mathbf{M}\right\}$. This in turn means that all selection functions for a finite theory $A$ are (')-covering and in fact full. They are not only complete ( $A$-elementary) but even elementary (cf. [1, Observation 4.6]).

§4. Postulates for contraction functions. We now turn to a set of rationality criteria which has gained some prominence in the literature on belief change. The basic $A G M$ postulates are given by $(-1)-(-6)$, and the two supplementary ones are $(-7)$ and $(-8)$. For their motivation, see [5].

$(-1) A-x$ is a theory.

$(-2) A-x \subseteq A$.

$(\div 3)$ If $x \notin A$, then $A-x=A$.

$(-4)$ If $x \in A-x$, then $\vdash x$.

$(-5) A \subseteq C n((A-x) \cup\{x\})$.

$(\dot{-6)}$ If $C n(x)=C n(y)$, then $A-x=A-y$.

$(-7) A-x \cap A-y \subseteq A \dot{-}(x \wedge y)$

$(-8)$ If $x \notin A-(x \wedge y)$, then $A-(x \wedge y) \subseteq A-x$.

These postulates, and all following postulates. are understood as quantified over all theories $A$ and all propositions $x$ and $y$. It follows from $(-1)$ and $(\dot{-5})$ that $A-x=A$ for every $x \in C n(\varnothing)$. We introduce two new conditions.

$(-8 \mathrm{r}) \quad A-(x \wedge y) \subseteq C n(A-x \cup A \dot{-})$.

$(\div 8 \mathrm{c})$ If $y \in A-(x \wedge y)$, then $A-(x \wedge y) \subseteq A \div x$.

With two very recent exceptions, ${ }^{5}$ I have never seen a condition like $(-8 \mathrm{r})$ discussed in writings on the logic of theory change. Given $(-4),(-8)$ implies the "covering condition"

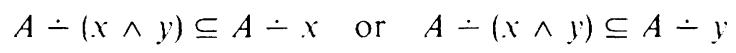

$[1$, Observation 3.4$]$ and hence $(-8 \mathrm{r}){ }^{6}$ Postulate $(-8 \mathrm{c})$ was found to be relevant in [22], where it has the same name. The " $r$ " in $(-8 r)$ stands for "relational", and "c" stands for "cumulative". The first name will be explained by the present paper, the second one is explained in [22]. For contraction functions satisfying $(-4)$,

${ }^{5}$ Both were discovered independently and concern belief revision rather than belief contraction. The first exception is condition (R.9) in [11]. The second is the infinitary condition "Gamma" in [16] which is labelled $(B C 7)$ in its variant for belief revision operations.

${ }^{6}$ Incidentally, it is proved in $[1$, Observation 6.5$]$ that the conjunction of $(-7)$ and $(-8)$ is equivalent to the even stronger "ventilation condition"

$$
A-(x \wedge y)=A-x \text { or } A-(x \wedge y)=A-y \text { or } A-(x \wedge y)=A-x \cap A-y
$$




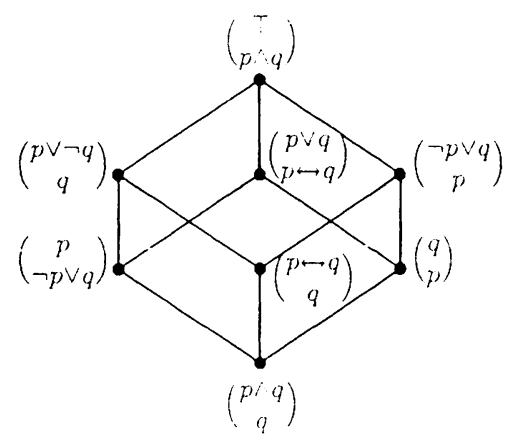

Figure 1

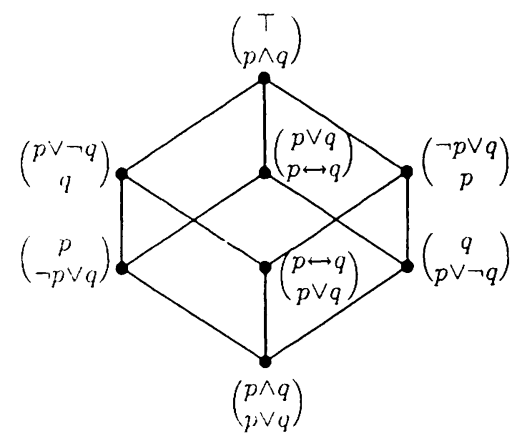

Figure 2

$(-8 \mathrm{c})$ is also a weakening of $(-8)$. However, there is no logical relationship between $(-8 \mathrm{c})$ and $(-8 \mathrm{r})$, not even in the finite case and in the presence of $(-1)-$ $(-7)$. To see this, consider the propositional language $L$ over the two atoms $p$ and q. In the following two figures, " $\left(\begin{array}{l}x \\ y\end{array}\right)$ " is short for " $A-x=C n(y)$ ". It is easily verified that the contraction function - over $A=C n(p \wedge q)$ defined in Figure 1 satisfies $(-1)-(-7)$ and $(-8 \mathrm{r})$, but it does not satisfy $(-8 \mathrm{c})$, because $q \in A \dot{-}(p \wedge q)$ but $C n(q)=A-(p \wedge q) \nsubseteq A \dot{-1}=C n(\neg p \vee q)$. On the other hand, the contraction function - over the same theory $A$ defined in Figure 2 satisfies $(-1)-(-7)$ and $(-8 \mathrm{c})$, but it does not satisfy $(-8 \mathrm{r})$, because

$$
\begin{aligned}
C n(p \vee q) & =A-(p \wedge q) \quad \nsubseteq \quad C n((A-p) \cup(A-q)) \\
& =C n(\{\neg p \vee q, p \vee \neg q\})=C n(p \leftrightarrow q) .
\end{aligned}
$$

We now relate the abstract postulates for contraction functions to our previous requirements for selection functions in partial meet contraction.

Lemma 7. Let $A$ be a theory. If $\dot{-}=\mathscr{C}(;)$ for some selection function $;$ for $A$ and

$\begin{aligned} \gamma \text { satisfies } & \left\{\begin{array}{l}\text { (a) }-, \\ \text { (b) }\left(\mathrm{I}^{\prime \prime}\right), \\ \text { (c) }\left(\mathrm{II}^{\prime \prime}\right) \text { and } ; \text { is complete, } \\ \text { (d) }\left(\mathrm{III}^{\prime \prime}\right), \\ \text { (e) }\left(\mathrm{IV}^{\prime \prime}\right),\end{array}\right\} \\ \text { then }- \text { satisfies } & \left\{\begin{array}{l}\text { (a) }(-1)-(-6), \\ \text { (b) }(-1)-(-7), \\ \text { (c) }(-1)-(-6) \text { and }(-8 \mathrm{r}), \\ \text { (d) }(-1)-(-6) \text { and }(-8 \mathrm{c}), \\ \text { (e) }(-1)-(-6) \text { and }(-8) .\end{array}\right\}\end{aligned}$

PROOF. Let - be a partial meet contraction function over $A$ determined by $;$.

(a) It is proved in $[1$, Observation 2.5$]$ that - satisfies $(-1)-(-6)$. It is easy to verify that $(-7)$ and $(-8)$, and thus also $(-8 \mathrm{r})$ and $(-8 \mathrm{c})$, are satisfied whenever 
one of the limiting cases $x \notin A-\operatorname{Cn}(\varnothing)$ or $y \notin A-C n(\varnothing)$ holds. In the rest of this proof, we always presume the principal case $x, y \in A-\operatorname{Cn}(\varnothing)$.

(b) Now let $;$ satisfy (I"), i.e., let $;(A \perp(x \wedge y)) \subseteq \gamma(A \perp x) \cup \gamma(A \perp y)$. Hence, $\bigcap(\gamma(A \perp x)) \cap \bigcap(;(A \perp y))=\bigcap(;(A \perp x) \cup ;(A \perp y)) \subseteq \bigcap ;(A \perp(x \wedge y))$, i.e., $A-x \cap A-y \subseteq A-(x \wedge y)$. That is, - satisfies $(\dot{-})$.

(c) Now let $\gamma$ be complete and satisfy (II"). Let $z \in A \dot{-}(x \wedge y)=\bigcap \gamma(A \perp(x \wedge y))$. So by (II"),$z \in \bigcap(\gamma(A \perp x) \cap i(A \perp y))$. Now suppose for reductio that $z \notin$ $C n(A-x \cup A-y)$. Then there is an $M \in A \perp z$ such that $(\bigcap \gamma(A \perp x)) \cup$ $(\bigcap \gamma(A \perp y)) \subseteq M$. Since $\gamma$ is complete, we get $M \in \gamma(A \perp x)$ and $M \in \gamma(A \perp y)$, so $M \in \gamma(A \perp x) \cap \gamma(A \perp y)$. But $z \notin M$, so $z \notin \bigcap(\gamma(A \perp x) \cap \gamma(A \perp y))$ which gives us a contradiction. So - satisfies $(-8 \mathrm{r})$.

(d) Now let $\gamma$ satisfy (III"). Since the antecedents of $\left(\right.$ III $\left.^{\prime \prime}\right)$ and $(-8 \mathrm{c})$ are identical and $\gamma(A \perp x) \subseteq \gamma(A \perp(x \wedge y))$ entails $\bigcap \gamma(A \perp(x \wedge y)) \subseteq \bigcap \gamma(A \perp x)$, it is obvious that $\dot{-}$ satisfies $(-8 \mathrm{c})$.

(e) Now let $\gamma$ satisfy $\left(I^{\prime \prime}\right)$. By exactly the same argument as in (d), - satisfies $(-8)$.

Given a contraction function - over $A$, we can derive from it a selection function $\gamma=$ for $A$. The idea is that an element of $A \perp x$ is a best element of $A \perp x$ if it includes everything which is included in the contraction of $A$ with respect to $x$. So we define $\gamma-(A \perp x)$ as $\{M \in A \perp x: A-x \subseteq M\}$, for every $x \in A-C n(\varnothing)$. Instead of $\hat{\gamma}$. we shall also write $\mathscr{S}(\dot{-})$.

Lemma 8. Let $A$ be a theory. If - is a contraction function over $A$ satisfying

$$
\left\{\begin{array}{l}
\text { (a) }(-1)-(-6), \\
\text { (b) }(-1)-(-7), \\
\text { (c) }(-1)-(-6) \text { and }(-8 \mathrm{r}), \\
\text { (d) }(-1)-(-6) \text { and }(-8 \mathrm{c}), \\
\text { (e) }(-1)-(-6) \text { and }(-8),
\end{array}\right\}
$$

then - is a partial meet contraction function determined by the selection function $\mathscr{S}(\dot{-})$, i.e., $\dot{-}=\mathscr{C}(\mathscr{S}(\dot{-}))$, and $\mathscr{P}(\dot{-})$ is complete

and satisfies

$$
\left\{\begin{array}{ll}
\text { (a) } & -, \\
\text { (b) } & \left(\mathrm{I}^{\prime \prime}\right) \\
\text { (c) } & \left(\mathrm{II}^{\prime \prime}\right) \\
\text { (d) } & \left(\text { III') }^{\prime \prime}\right) \\
\text { (e) } & \left(\text { IV }^{\prime \prime}\right)
\end{array}\right\}
$$

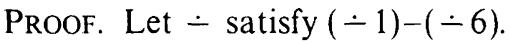

(a) It is proved in [1, Observation 2.5], that $\mathscr{P}(\dot{-})$ is a selection function for $A$ and that $\dot{-}$ is determined by $\mathscr{S}(\dot{-})$. We add the check for well-definedness. In the principal case $x, y \in A-C n(\varnothing), A \perp x=A \perp y$ entails that $C n(x)=C n(y)$; for if it were the case that $x \forall y$, say, then there would be an element in $A \perp y$ containing $x$, contrary to $A \perp x=A \perp y$. But from $C n(x)=C n(y)$ and $(-6)$ it follows by the 
definition of $\mathscr{S}(\dot{-})$ that $\gamma(A \perp x)=i(A \perp y)$, as desired. By construction, $\mathscr{Y}(\dot{-})$ is

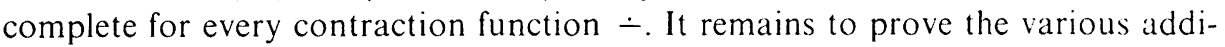
tional properties of $\mathscr{F}(\dot{-})$.

(b) Let $\dot{-}$ in addition satisfy $(-7)$, and let $M \in ; \div(A \perp(x \wedge y))$. We want to

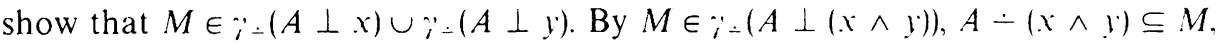
so by $(-7), A-x \cap A \dot{-} \subseteq \subseteq M$. Now suppose for reductio that $M \notin ; \dot{\sim}(A \perp x) \cup$ $\gamma \cdot(A \perp y)$, i.e., neither $A-x \subseteq M$ nor $A-y \subseteq M$. Take $z \in(A-x)-M$ and $z^{\prime} \in(A \dot{-y})-M$. By $z, z^{\prime} \in A, z, z^{\prime} \notin M$, and the primeness of all elements of $U_{A}$, $z \vee z^{\prime} \notin M$. But on the other hand, since $z \vee z^{\prime} \in A \dot{-} \cap \cap A \dot{-} \subseteq \subseteq M$, we get $z \vee z^{\prime} \in M$, so we have a contradiction. So $\mathscr{P}(\dot{-})$ satisfies $\left(\mathrm{I}^{\prime \prime}\right)$.

(c) Now let - satisfy $(-1)-(-6)$ and $(-8 \mathrm{r})$, and let $M \in ; \div(A \perp x) \cap ; \div(A \perp y)$. We want to show that $M \in ;-(A \perp(x \wedge y))$. But as our hypothesis means that $A-x \cup A-y \subseteq M$ and $M$ is a theory, we have $C n(A-x \cup A-y) \subseteq M$. so by $(-8 \mathrm{r}) A-(x \wedge y) \subseteq M$, so $M \in i^{\prime}(A \perp(x \wedge y))$. So $\mathscr{P}(\dot{-})$ satisfies $\left(\mathrm{II}^{\prime \prime}\right)$.

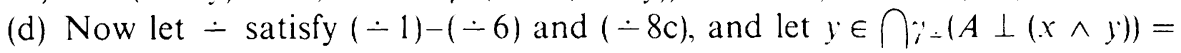
$A-(x \wedge y)$ and $M \in \imath^{\circ}(A \perp x)$. The latter condition means that $A-x \subseteq M$, so by $(-8 \mathrm{c}) A-(x \wedge y) \subseteq M$, so $M \in \gamma \cdot(A \perp(x \wedge y))$, as desired. So $\mathscr{\mathscr { T }}(\dot{-})$ satisfies (III").

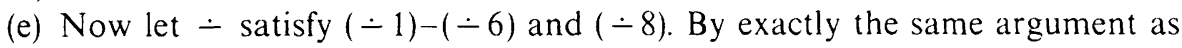
the last one, $\mathscr{P}(-)$ satisfies $\left(I^{\prime \prime}\right)$.

§5. Representation theorems for contraction functions. We get four representation theorems for partial meet contraction as a result of conjoining previously established lemmas. Our Theorems 1 and 4 are proven in [1] although our construction for obtaining the latter appears to be quite different from the one used there. Theorems 2 and 3 are new; the "completeness" half of the former is generally valid, the remainder of the new results hold only under various additional conditions. All these conditions are satisfied if $A$ is finite. It should be noted that our proofs of the completeness halves use one and the same method in that they all work with Samuelson revealed preferences.

THEOREM 1. A contraction function - over A satisfies $(-1)-(-6)$ if and only if it is a partial meet contraction function.

ProOF. See Lemma 7 (a) and 8 (a) above, according to which in the cases indicated $\mathscr{C}(\gamma)$ satisfies $(-1)-(-6)$ and $\mathscr{P}(-)$ is a selection function for $A$ such that $\mathscr{C}(\mathscr{P}(\dot{-}))=\dot{-}$. (Theorem 1 is Observation 2.5 of [1].)

It is clear from Lemma 8 that in the proof of the completeness half of Theorem 1 the determining selection function is chosen complete, but we do not think it is necessary to state this in the theorem. The same comment applies to the following three representation theorems.

THEOREM 2. Every relational partial meet contraction function - over A satisfies $(-1)-(-7)$, and if $\dot{-}$ is determined by a selection function that is both relational and complete (equivalently, A-elementary), then it satisfies $(-8 \mathrm{r})$. Conversely, every contraction function - over A satisfying $(-1)-(-7)$ and $(-8 \mathrm{r})$ is a relational partial meet contraction function.

Proof. For the first part, let - be a partial meet contraction function determined by a preference relation $\leq$. By Lemma $1($ a), $\mathscr{T}(\leq)$ satisfies (I) and (II). Since $A \perp$ is subtractive, it also satisfies $\left(\mathrm{I}^{\prime}\right)$ and $\left(\mathrm{II}^{\prime \prime}\right)$, by Lemma $2(\mathrm{a})$, and also ( $\left.\mathrm{I}^{\prime \prime}\right)$ and 
$\left(\mathrm{II}^{\prime \prime}\right)$, by reformulation. So by Lemma $7(\mathrm{~b}), \mathscr{C}(\mathscr{P}(\leq))$ satisfies $(-1)-(-7)$, and by Lemma $7(\mathrm{c})$, it satisfies $(-8 \mathrm{r})$, if $\mathscr{P}(\leq)$ is complete, i.e., $\Delta$-elementary.

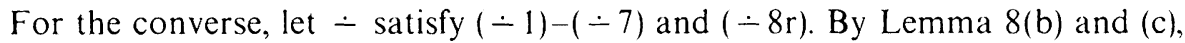
$\mathscr{C}(\mathscr{S}(-))=-$ and $\mathscr{S}(-)$ satisfies $\left(\mathrm{I}^{\prime \prime}\right)$ and $\left(\mathrm{II}^{\prime \prime}\right)$, and also $\left(\mathrm{I}^{\prime}\right)$ and $\left(\mathrm{II}^{\prime}\right)$, by reformulation. Since $A \perp$ is subtractive, $\mathscr{P}(-)$ satisfies (I), by Lemma 4(a). Since $A \perp$ is also finitely additive and compact, $\mathscr{S}(\dot{-})$ is relational with respect to $\mathscr{P}(\mathscr{S}(\dot{-}))$, by Lemma $4($ b). That is, $\mathscr{S}(-)=\mathscr{S}(\mathscr{P}(\mathscr{P}(\dot{-})))$. Hence, $-=\mathscr{C}(\mathscr{S}(\mathscr{P}(\mathscr{S}(\dot{-}))))$, i.e., $\dot{-}$ is relational with respect to $\mathscr{P}(\mathscr{P}(\dot{-}))$.

The completeness of $\gamma$ is necessary in the "soundness" part of Theorem 2. The possible failure without completeness is due to the fact that for sets $\mathbf{W}^{\prime}$ and $\mathbf{W}^{\prime \prime}$ of maximally consistent sets of propositions, it does not necessarily hold that $\bigcap\left(\mathbf{W}^{\prime} \cap \mathbf{W}^{\prime \prime}\right) \subseteq C n\left(\bigcap \mathbf{W}^{\prime} \cup \bigcap \mathbf{W}^{\prime \prime}\right)$.

THEOREM 3. Every relational partial meet contraction function - over $A$ determined by a smooth and negatively transitive preference relation satisfies $(-1)-(-7)$ and $(-8 \mathrm{c})$. In partial converse, every contraction function - over $A$ satisfying $(-1)-$ $(-7),(-8 \mathrm{r})$, and $(-8 \mathrm{c})$ is a relational partial meet contraction function, and if $A \perp$ is 123-covering, then the determining preference relation can be chosen negatively transitive.

Proof. For the first part, let - be a partial meet contraction function determined by a smooth and negatively transitive preference relation $\leq$. We show in the same way as in the proof of Theorem 2 that $\mathscr{P}(\leq)$ satisfies (I') and (II'), and we know from Lemma $3($ b) and Lemma $4(\mathrm{c})$ that it satisfies (III) and (III'). So by Lemma $7(\mathrm{~b})$ and $(\mathrm{d}), \mathscr{C}(\mathscr{S}(\leq))$ satisfies $(-1)-(-7)$ and $(-8 \mathrm{c})$.

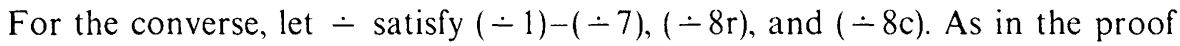
of Theorem 2 , we get that $\dot{-}$ is relational with respect to $\mathscr{P}(\mathscr{S}(\dot{-}))$. Moreover, by Lemma $8(\mathrm{~b}), \mathscr{S}(-)$ satisfies $\left(\mathrm{I}^{\prime}\right)$, and by Lemma $8(\mathrm{~d}), \mathscr{S}(-)$ satisfies $\left(\right.$ III $\left.^{\prime}\right)$ and also (III), by Lemma 4 (c). Hence, if $A \perp$ is 123 -covering, then $\mathscr{P}(\mathscr{S}(-))$ is negatively transitive, by Lemma 2(b).

THEOREM 4. A contraction function - over $A$ satisfies $(-1)-(-6),(-7)$, and $(-8)$ if and only if it is a transitively relational partial meet contraction function.

Proof. This is Observation 4.4 of [1], but we shall sketch our proof which is quite different from the construction offered there.

For the first part of the theorem, let - be a partial meet contraction function determined by a transitive preference relation $\leq$. We show in the same way as in the proof of Theorem 2 that $\mathscr{P}(\leq)$ satisfies $\left(\mathrm{I}^{\prime}\right)$ and $\left(\mathrm{II}^{\prime}\right)$, and we know from Lemma $3(\mathrm{c})$ and Lemma $4(\mathrm{~d})$ that it satisfies (IV) and $\left(\mathrm{IV}^{\prime}\right)$. So by Lemma 7 (b), $\mathscr{C}(\mathscr{S}(\leq))$ satisfies $(-1)-(-7)$, and by Lemma $7(\mathrm{e})$, it satisfies $(-8)$.

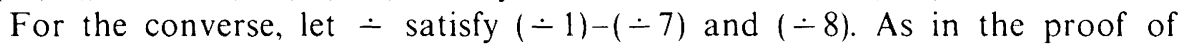
Theorem 2 , we get that - is relational with respect to $\mathscr{P}(\mathscr{P}(-))$. Moreover, by Lemma 8(e), $\mathscr{P}(-)$ satisfies (IV') and also (IV), by Lemma 4(d). Since $A \perp$ is finitely additive, $\mathscr{P}(\mathscr{S}(\dot{-}))$ is transitive, by Lemma $2(\mathrm{c})$.

COROLlaRY 2. Let $A$ be a logically finite theory:

Then - is a

$$
\left\{\begin{array}{c}
-, \\
\text { relational, } \\
\text { negatively transiticely relational, } \\
\text { transitively relational, }
\end{array}\right\}
$$


partial meet contraction function iff it satisfies

$$
\left\{\begin{array}{c}
(-1)-(-6), \\
(-1)-(-7) \text { and }(-8 \mathrm{r}), \\
(-1)-(-7),(-8 \mathrm{r}), \text { and }(-8 \mathrm{c}) \\
(-1)-(-7) \text { and }(-8) .
\end{array}\right\}
$$

Proof. By Theorems $1-4$, because for finite theories $A, \gamma$ over $A \perp$ is complete, every negatively transitive nonstrict preference relation $\leq$ over $A$ is smooth, and $A \perp$ is 123 -covering.

It is easy to locate the impact of the finiteness assumption. In the case of relational partial meet contractions (Theorem 2), it is a constraint (completeness) imposed by model theory which has to be met in order to make the "soundness" direction work. In the case of negatively transitively relational partial meet contractions (Theorem 3), it is a constraint imposed by the theory of rational choice (that $\gamma$ be 123-covering and smooth) which is satisfied without strains on intuitive plausibility only in the finite case.

It should be noted that although $(-8)$ in the context of the other postulates implies $(-8 \mathrm{r})$ and $(-8 \mathrm{c})$, transitive relationality does not imply negatively transitive relationality. However, transitivity in companion with connectivity implies negative transitivity. And it is known from $[1, \S 5]$ that a connectivity requirement on the underlying preference relation changes very little in the partial meet mechanism. ${ }^{7}$

We conclude this section with a direct representation of the Samuelson preferences over $U_{A}$ revealed by contraction behavior. Let $\leq=\mathscr{P}(\mathscr{S}(\dot{-}))$. Then in the general case, $M \leq M^{\prime}$ iff there is an $x$ such that $A-x \subseteq M^{\prime}$ and $M \in A \perp x$, or there is no $x$ such that $A-x \subseteq M$. We recall, for contrast, the construction used in the proof of [1, Observation 4.4]. There $M \leq M^{\prime}$ is defined to hold between sets $M$ and $M^{\prime}$ from $U_{A}$ iff for all $x$ such that $A-x \subseteq M$ and $M^{\prime} \in A \perp x$, it holds that $A-x \subseteq M^{\prime}$, and there is an $x \in A$ such that $A-x \subseteq M^{\prime} .{ }^{8}$ Evidently, this definition is quite different from the one we used, and it is doubtful whether it is suitable for any case but the strongest one which is treated in Theorem 4. It is interesting, however, that AGM employ our construction in their proof of Observation 4.10 of [1]. In the finite case, it is not difficult to find out that according to our definition, $M \leq M^{\prime}$ iff $A-\bar{m} \wedge \overline{m^{\prime}} \subseteq M^{\prime}$, or equivalently (!), iff $\overline{m^{\prime}} \notin A \sqcup \bar{m} \wedge \overline{m^{\prime}}$.

§6. The logic of prioritized base contractions in the finite case. In this section we present the concept of prioritized base contraction as studied in particular by Nebel [18], [19]. On the face of it, these belief change operations are entirely different from the partial meet contractions discussed so far. As Nebel has shown, however, very close connections can be established between the two approaches. We shall further elaborate this theme and determine the logic of prioritized base contrac-

\footnotetext{
'In order to reconcile this with your intuitions, cf. footnote 1.

${ }^{8}$ This simplified rephrasing of the AGM construction makes use of the fact that for all $M \in U_{A}$ and contraction functions - satisfying the AGM postulates, $A-x \subseteq M$ implies $M \in A \perp x$.
} 
tions in terms of the above-mentioned postulates by applying one of our central representation theorems.

Let $B$ be a finite set of propositions. $B$ is called a (simple) belief base for the theory $A$ if $C n(B)=A$. B is called prioritized if there is a weak ordering, i.c., a transitive and connected relation $\preceq$ over $B$ (Nebel's [18]. [19] "epistemic relevance"). The relation $\sim=\preceq \cap \preceq^{-1}$ partitions $B$ into finitely many equivalence classes $B_{1}, \ldots, B_{n}$. We label the classes in such a way that $x_{i} \leq x_{j}$ for $x_{i} \in B_{i}$ and $x_{j} \in B_{j}$ with $i \leq j$. Generalizing slightly, we say that a prioritized belief base for $A$ is a finite sequence $\left\langle B_{1}, \ldots, B_{n}\right\rangle$, or $\left\langle B_{i}\right\rangle$ for short, of finite sets of propositions such that $C n\left(\cup B_{i}\right)=A$. Simple belief bases are now special cases of prioritized belief bases with $n=1$. We do not require that the $B_{i}$ 's are disjoint or mutually consistent. Intuitively, the propositions in $B_{n}$ are the most important (most relevant, most valuable, most certain, etc.) pieces of information, and the propositions in $B_{1}$ are the least important. One can think of the complement of $\bigcup B_{i}$ as the set $B_{0}$ of propositions for which there is no independent evidence. Although the finiteness of $B=\bigcup B_{i}$ does not ensure that the generated theory $A$ is logically finite, our central result in this section, Theorem 7, will be restricted to this case.

Prioritized base contractions are contractions of theories $A$ presented in the form of (generated by, associated with) a fixed prioritized belief base $\left\langle B_{i}\right\rangle$. The basic idea is to minimize the loss of propositions at every level of "priority".

For every $i \in\left\{1, \ldots, n_{\}}\right.$, let $B_{\geq i}$ be the set of all elements of $B=\bigcup B_{i}$ with a priority of at least $i$, i.e., $B_{\geq i}=\bigcup\left\{B_{j}: j \geq i\right\}$. Furthermore, let $C_{i}=C \cap B_{i}$ and $C_{\geq i}=C \cap B_{\geq i}$ for any set of propositions $C$. Then, in the context of this section, $C$ is a preferred $x$-discarding subset of $B$ if $C \subseteq B$ and for every $i, C_{\geq i}$ is an inclusion maximal subset of $B_{\geq i}$ subject to the condition that $x$ is not implied.

Two kinds of information are used in prioritized base contraction: the syntactical information encoded in the structure of the propositions in $B$, and the weighting of these propositions expressed by $\preceq$. If the belief base is simple, i.e., if $n=1$, then we only exploit syntactical information. Prioritized base contractions and revisions are studied in an infinitistic framework by Nebel [18], [19]. ${ }^{9}$ He shows that prioritized base revisions can be represented by partial meet theory revisions. Then he proves that a certain variant of simple base contraction satisfies $(-1)-(-7)$ but not $(-8)$, and a corresponding result for prioritized base revisions. However, Nebel leaves open the question which logic is characterized by prioritized base contractions. Building on Theorem 3 and its corollary, we shall answer this question for the finite case. Our concern will be the slightly more complicated case of prioritized base contractions, and we try to make our proof more transparent by introducing the concept of a base-oriented selection function.

Now consider the following strict preference relations between arbitrary sets of propositions. For every $i \in\{1, \ldots, n\}$ and any two sets $C$ and $C^{\prime}$, we write $C \prec \prec{ }_{i} C^{\prime}$

\footnotetext{
${ }^{9}$ Nebel's treatment of the fully general infinite case in [19] is not quite correct. Slips have crept into his claim that his $C \Downarrow \phi$ is nonempty, into his definition (9) of $\ll$, and into the proof of Proposition 8. As Nebel (personal communication) has suggested, they can be remedied by imposing a condition of converse well-foundedness on $\preceq$.
} 
if and only if $C_{i} \subset C_{i}^{\prime}$ and $C_{j} \subseteq C_{j}^{\prime}$ for every $j>i$. We write $C \prec \prec C^{\prime \prime}$ if there is an $i$ such that $C \prec \prec_{i} C^{\prime}$. The relation $\prec \prec$ is to reflect the fact that intuitively a set $C^{\prime}$ is better than another set $C$ just in case it contains more important beliefs than $C$. In particular, $\prec \prec$ satisfies a version of Hansson's [9] maximizing property, because $C \cap B \subset C^{\prime} \cap B$ implies $C \prec \prec C^{\prime}$. It is immediately verified that $C$ is a preferred $x$-discarding subset of $B$ if and only if it is a maximal element in $B \perp x$ under $\prec \prec$. (Recall that $B \perp x$ is the set $\{N \subseteq B: x \notin C n(N)$ and $x \in C n(D)$ for all $D$ with $N \subset D \subseteq B\}$.)

Following Nebel, we let $\preceq \prec$ be the converse complement of $\prec \prec$, i.e., $C \longleftrightarrow \prec C^{\prime}$ iff not $C^{\prime} \prec \prec C$. We denote this preference relation over arbitrary sets of propositions by $\mathscr{P}\left(\left\langle B_{i}\right\rangle\right)$. Clearly, since $\prec \prec$ is irreflexive, asymmetric, conversely well-founded, and transitive, $\mathscr{P}\left(\left\langle B_{i}\right\rangle\right)$ is reflexive, connected, negatively well-founded, and negatively transitive. But $\mathscr{P}\left(\left\langle B_{i}\right\rangle\right)$ is not transitive, not even in the special case of simple belief bases $(n=1)$ where $\preceq \prec$ coincides with $\not \supset$.

As before, a selection function $\gamma=\mathscr{Y}^{\prime}(\preceq \prec)$ can be defined by stringent maximization. But this time $\gamma$ is a selection function for the base $B$ as well as for the theory $A$, and its domain may in fact be construed as the class of all nonempty sets of sets propositions in $L$, that is $2^{2^{L}}-\{\varnothing\}$. In the following, it is understood that $;(B \perp x)=\left\{N \in B \perp x: N^{\prime} \preceq \prec N\right.$ for all $\left.N^{\prime} \in B \perp x\right\}$, while $\gamma(A \perp x)=$ $\left\{M \in A \perp x: M^{\prime} \prec \prec M\right.$ for all $\left.M^{\prime} \in A \perp x\right\}$. Either way, $\gamma$ is a selection function in the sense of subsection 2.1 .

Let $\left\langle B_{i}\right\rangle$ be a prioritized belief base for $A$, and let $i=\mathscr{P}\left(\mathscr{P}\left(\left\langle B_{i}\right\rangle\right)\right)$ be the selection function over $A \perp$ determined by $\left\langle B_{i}\right\rangle$. A straightforward idea to get prioritized base contractions is the following:

$$
A-x= \begin{cases}\bigcap\left\{\operatorname{Cn}(N): N \in \gamma^{\prime}(B \perp x)\right\} & \text { for every } x \in A-\operatorname{Cn}(\varnothing), \\ A & \text { for every } x \notin A \text { and every } x \in \operatorname{Cn}(\varnothing) .\end{cases}
$$

In the special case of simple belief bases $(n=1)$ we have $;(B \perp x)=B \perp x$, so this definition boils down to the full meet $\bigcap\{B \perp x\}$.

Contraction functions obtained in this way satisfy most but not all of the postulates we would want them to satisfy.

THEOREM 5. If - is a prioritized base contraction function as determined by the above definition, then it satisfies $(-1)-(-4),(-6),(-7)$, and $(-8 \mathrm{c})$. However, even if the base is simple, - will in general fail to satisfy $(-5)$ and $(-8 \mathrm{r})$.

Proof. It is obvious that - satisfies $(-1)-(-4)$ and $(-6)$.

For $(-7)$, assume that $N \vdash z$ for every $N \in ;(B \perp x) \cup i(B \perp y)$. We need to show that $N^{\prime} \vdash z$ for every $N^{\prime} \in ;(B \perp(x \wedge y))$. Let $N^{\prime} \in ;(B \perp(x \wedge y)) \subseteq B \perp(x \wedge y)$. First, we note that every element of $B \perp(x \wedge y)$ is either in $B \perp x$ or in $B \perp y$. Without loss of generality, assume that $N^{\prime} \in B \perp x$. We are ready if we can show that $N^{\prime}$ is in ${ }^{\prime}(B \perp x)$. Suppose it is not. Then there is an $N_{1} \in B \perp x$ such that $N^{\prime} \prec \prec N_{1}$. $N_{1}$ must not be in $B \perp(x \wedge y)$, since $N^{\prime}$ is in $\gamma^{\prime}(B \perp(x \wedge y))$. Because $N_{1} \in B \perp x-$ $B \perp(x \wedge y)$, we get that there is a proper superset $N_{2}$ of $N_{1}$ such that $N_{2} \in B \perp y$. Since $N_{1} \in B \perp x, N_{2} \vdash x$. Since every proper superset of $N_{2}$ satisfies both $x$ and $y$, $N_{2}$ is in $B \perp(x \wedge l)$. By the maximizing property, we have $N_{1} \prec \prec N_{2}$. By the transitivity of $\prec \prec$, we get $N^{\prime} \prec \prec N_{2}$. This, however, contradicts $N^{\prime} \in ;(B \perp(x \wedge y))$ and $N_{2} \in B \perp(x \wedge y)$. Therefore, our supposition must have been false. 
For $(-8 \mathrm{c})$, assume that, first, $N \vdash y$ and, second, $N \vdash z$ for every $N \in ;(B \perp(x \wedge y))$. We need to show that $N^{\prime} \vdash z$ for every $N^{\prime} \in \gamma^{\prime}(B \perp x)$. Let $N^{\prime} \in \gamma(B \perp x)$, and suppose for reductio that $N^{\prime} H z$. If $N^{\prime}$ is in $B \perp(x \wedge y)$ then there must be, by the second assumption, an $N_{1}$ in $B \perp(x \wedge y)$ such that $N^{\prime} \prec \prec N_{1}$. In fact, $N_{1}$ can be chosen from $\gamma(B \perp(x \wedge y))$, by the transitivity and converse well-foundedness of $\prec \prec$. By the first assumption, $N_{1} \in B \perp x$. But this contradicts $N^{\prime} \prec<N_{1}$ and $N^{\prime} \in \gamma(B \perp x)$. Hence, $N^{\prime}$ cannot be in $B \perp(x \wedge y)$. But because $N^{\prime} \mid x \wedge y$, there must be a proper superset $N_{2}$ of $N^{\prime}$ in $B \perp(x \wedge y)$. We know that $N_{2} \vdash x$, by $N^{\prime} \in$ $B \perp x$. By the first assumption then, $N_{2}$ cannot be in $;(B \perp(x \wedge y))$. By the transitivity and converse well-foundedness of $\prec \prec$, there must be an $N_{3}$ in $;(B \perp(x \wedge y))$ such that $N_{2} \prec \prec N_{3}$. By our first assumption, $N_{3}$ is in $B \perp x$. By the maximizing property, we have $N^{\prime} \prec \prec N_{2} \prec \prec N_{3}$, so by transitivity $N^{\prime} \prec \prec N_{3}$. This, however, contradicts $N^{\prime} \in \gamma(B \perp x)$ and $N_{3} \in B \perp x$. Therefore, our supposition must have been false.

The failure of $(-5)$ is obvious. Consider for instance $B=\{p \wedge q\}$, for which $A=C n(p \wedge q) \nsubseteq C n((A-p) \cup\{p\})=C n(p)$.

The failure of $(-8 \mathrm{r})$ is not so easy to see. The simplest examples involve three atoms $p, q, r$ and look somewhat like the following. Let $B$ consist of the following four propositions:

$$
\begin{aligned}
(p \wedge q) \vee(p & \wedge r) \vee(q \wedge r), \quad r \rightarrow(p \leftrightarrow q), \\
q & \wedge(p \leftrightarrow r), \quad p \wedge r .
\end{aligned}
$$

Clearly, $A=C n(B)=C n(p \wedge q \wedge r)$. Note that $q \wedge(p \leftrightarrow r)$ implies $r \rightarrow(p \leftrightarrow q)$ and that $p \wedge r$ implies $(p \wedge q) \vee(p \wedge r) \vee(q \wedge r) .(p \wedge q) \vee(p \wedge r) \vee(q \wedge r)$ and $r \rightarrow(p \leftrightarrow q)$ taken together imply $p \wedge q$. This gives us

$$
B \perp p=\{\{(p \wedge q) \vee(p \wedge r) \vee(q \wedge r)\},\{r \rightarrow(p \leftrightarrow q), q \wedge(p \leftrightarrow r)\}\}
$$

and

$$
B \perp q=\left\{\{r \rightarrow(p \leftrightarrow q)\}_{j},\{(p \wedge q) \vee(p \wedge r) \vee(q \wedge r), p \wedge r\}\right\}
$$

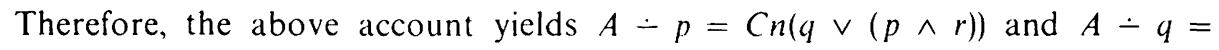
$C n(\neg(\neg p \wedge q \wedge r))$, so $C n(A-p \cup A-q)=C n((p \wedge r) \vee(q \wedge \neg r))$. On the other hand,

$$
B \perp(p \wedge q)=\left\{\{p \wedge q) \vee(p \wedge r) \vee(q \wedge r), p \wedge r_{\}},\{r \rightarrow(p \leftrightarrow q), q \wedge(p \leftrightarrow r)\}\right\},
$$

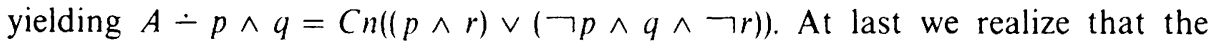
union of $A-p$ and $A-q$ does not imply $(p \wedge r) \vee(\neg p \wedge q \wedge \neg r)$, because the former but not the latter is compatible with $p \wedge q \wedge \neg r$.

In so far as one considers the postulates $(-5)$ and $(-8 \mathrm{r})$ to be plausible principles of theory change - which in fact we do--Theorem 5 shows that the above definition is defective. For this reason we introduce a slightly more sophisticated concept, and our official definition reads as follows. Let $a$ again be a representative of $A$.

The prioritized base contraction - over a logically finite theory $A$ determined by $\left\langle B_{i}\right\rangle$ is then given by 


$$
A-x= \begin{cases}\bigcap\left\{C n\left(N \cup\left\{x \rightarrow a_{\}}\right): N \in i(B \perp x)\right\}^{10}\right. & \text { for every } x \in A-C n(\varnothing), \\ A & \text { for every } x \notin A \text { and } x \in C n(\varnothing) .\end{cases}
$$

The singleton $\{x \rightarrow a\}$ is inserted in order to make sure that the recovery postulate $(-5)$ is satisfied. This trick is used by Nebel [18]. ${ }^{11}$ We shall see that this modification also solves our problem with $(-8 \mathrm{r})$. As a first indication of this, note that the official definition cures our above counterexample to $(-8 \mathrm{r})$ by strengthening $A-x$ to $C n(y \wedge(x \rightarrow z))$ and $A-y$ to $C n(y \rightarrow(x \wedge z))$, so that

$$
C n(A-x \cup A-y)=C n(x \wedge y \wedge z) \text {. }
$$

Since this has been the full theory $A,(-8 \mathrm{r})$ is clearly satisfied.

We denote the prioritized base contraction over $A$ determined by the prioritized belief base $\left\langle B_{i}\right\rangle$ by $\mathscr{C}\left(\left\langle B_{i}\right\rangle\right)$. A contraction function - over $A$ is a simple (or prioritized) base contraction function if there is a simple belief base $B$ (a prioritized belief base $\left\langle B_{i}\right\rangle$ ) for $A$ such that $\dot{-}=\left(6(B)\right.$ (respectively, $-=\mathscr{\gamma}\left(\left\langle B_{i}\right\rangle\right)$ ). In conformity with the terminology of $[1]$, simple base contractions could also be named full meet base contractions.

Lemma 9. Let $B=\bigcup B_{i}, A=C n(B), M \in A \perp x$, and let $;=\mathscr{Y}\left(\mathscr{P}\left(\left\langle B_{i}\right\rangle\right)\right)$. Then $M \in \gamma(A \perp x)$ iff $N \subseteq M$ for some $N \in i(B \perp x)$.

Let us call selection functions which harmonize choices in $A \perp$ with choices in $B \perp$ in the way exhibited in Lemma 9 base-oriented. The following result shows that the selection function $;$ induced by a prioritized belief base leads to the same result when applied directly to belief bases as when applied to the generated theory. Prioritized base contraction thus reduces to a special kind of partial meet theory contraction. A proof of almost the same fact has already been given by Nebel ( $[18$, Theorem 14] and [19, Theorem 7]). Our proof is somewhat more general in that it only turns on the fact that $i$ is base-oriented. Nothing hinges on the particular definition of $\prec \prec$.

THEOREM 6. Let $\left\langle B_{i}\right\rangle$ be a prioritized belief hase for a logically finite theory $A$, and let $\gamma=\mathscr{H}\left(\mathscr{P}\left(\left\langle B_{i}\right\rangle\right)\right)$. Then $\mathscr{\zeta}\left(\left\langle B_{i}\right\rangle\right)=\mathscr{C}\left(\eta_{i}\right)$.

Proof. For the principal case $x \in A-C n(\varnothing)$, we have to show that

$$
\bigcap\{C n(N \cup\{x \rightarrow a\}): N \in ;(B \perp x)\}=\bigcap_{i}(A \perp x) \text {. }
$$

By Lemma 9, we know that $;$ is base-oriented.

"LHS $\subseteq$ RHS": Let $N \cup\{x \rightarrow a\} \vdash y$ for every $N \in \eta_{i}(B \perp x)$, and let $M \in ;(A \perp x)$. Since $\gamma$ is base-oriented, there is an $N \in \gamma^{\prime}(B \perp x)$ such that $N \subseteq M$. So $M \cup$ $\{x \rightarrow a\} \vdash y$. But also, by the fullness of the elements in $A \perp x, x \rightarrow a \in M$ (see subsection 3.1). So $M \vdash y$, as desired.

"RHS $\subseteq$ LHS": Assume that there is an $N \in ;(B \perp x)$ such that $N \cup\{x \rightarrow a\}+y$. Hence, by the deduction theorem and supraclassicality, $N \cup\{y \rightarrow a\}+x$. Thus, there is an $M \in A \perp x$ such that $N \cup\{y \rightarrow a\} \subseteq M$. Since $N \subseteq M$ and $;$ is baseoriented, $M \in \gamma(A \perp x)$. Since $y \rightarrow a \in M, a \vdash x$ and $M \vdash x$, we have $y \notin M$. So $y \notin \bigcap \vartheta(A \perp x)$, as desired.

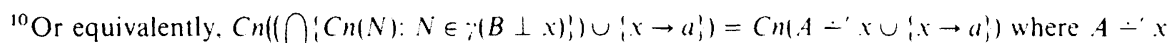
follows the first definition.

${ }^{11}$ Nebel's later paper by [19] deals with revisions where this problem does not arise. In fact. if revisions are construed as being generated by the so-called Levi-identity $A * x=\operatorname{Cn}(t A-\neg x) \cup(x$,$) , then$ the modification made in our official definition does not have any effect on revisions.
} 
We are now going to construct, for an arbitrary given preference relation $\leq$ over a logically finite theory $A$, an equivalent simple belief base $B$ for $A$. This base will be denoted $\mathscr{B}(\leq)$. For $M \in U_{A}$, let $\hat{M}$ be the set $\left\{M^{\prime} \in U_{A}: M^{\prime} \not \leq M\right\} \cup\{M\}$ of elements in $U_{A}$ which are not covered by $M$, together with $M$ itself. Let $\hat{m}$ be an abbreviation for the disjunction $\bigvee\left\{m^{\prime}: M^{\prime} \in \hat{M}\right\}$, with each $m^{\prime}$ being a representative of the respective $M^{\prime}$. Then $B=\mathscr{B}(\leq)$ is defined as the set $\left\{\hat{m}: M \in U_{A}\right\}$. Since $A$ is finite modulo $C n$, everything else is. This construction and the proof idea for the direction "(iii) $\Rightarrow$ (i)" in Theorem 7 is adapted from Lewis [15].

LEMMA 10. Let $A$ be a logically finite theory, and let $\leq$ be a reflexive and negatively transitive preference relation over $U_{A}$. Then $\left.\mathscr{P}().(\leq)\right)=\leq$.

Proof. As a preparatory consideration, let us consider what is contained in a set $M \cap B$, when $M \in U_{A}$ and $B=\mathscr{B}(\leq)$ for a given $\leq$. By definition, $M=C n(m)$. Now any proposition from $B$ is of the form $\widehat{m^{*}}$ for some $M^{*} \in U_{A}$ or, more explicitly, of the form $\bigvee\left\{m^{* *}: M^{* *} \in U_{A}\right.$, and $M^{* *} \not M^{*}$ or $\left.M^{* *}=M^{*}\right\}$. We know that a proposition $\widehat{m^{*}}$ from $B$ is in $M$ if and only if $m \vdash \widehat{m^{*}}$, i.e., $m \vdash$ $\bigvee\left\{m^{* *}: M^{* *} \in U_{A}\right.$, and $M^{* *} \not M^{*}$ or $\left.M^{* *}=M^{*}\right\}$. By Lemma 6(iii), this obtains just in case $M \not M^{*}$ or $M=M^{*}$.

Now let $\leq^{*}=\mathscr{P}(\mathscr{B}(\leq))$. We want to show that $M \leq^{*} M^{\prime}$ iff $M \leq M^{\prime}$. By definition, $M \leq^{*} M^{\prime}$ if and only if either $M \cap B \subseteq M^{\prime} \cap B$ or $M^{\prime} \cap B \nsubseteq M \cap B$. Applying our preparatory consideration, this means that either

(i) For every $M^{*} \in U_{A}$, if $M \not M^{*}$ or $M=M^{*}$, then $M^{\prime} \not M^{*}$ or $M^{\prime}=M^{*}$, or

(ii) There is an $M^{*} \in U_{A}$ such that $M^{\prime} \not \leq M^{*}$ or $M^{\prime}=M^{*}$, and $M \leq M^{*}$ and $M \neq M^{*}$.

We need to show that this is equivalent with $M \leq M^{\prime}$. That the latter implies (i) or (ii) is clear: If $M=M^{\prime}$, then (i), and if $M \neq M^{\prime}$, then (ii) is immediate by putting $M^{*}=M^{\prime}$.

For the converse, suppose that $M \not M^{\prime}$. We verify that this is incompatible with each of (i) and (ii). From (i), we get by putting $M^{*}=M$ that $M^{\prime} \not \leq M$ or $M^{\prime}=M$. In the former case we get from $M \not M^{\prime}$ and negative transitivity that $M \not M$, contradicting the reflexivity of $\leq$. The latter case does not fare better; it contradicts $M \not M^{\prime}$ as well. From (ii), we get that $M^{\prime} \not \leq M^{*}$ or $M^{\prime}=M^{*}$ for some $M^{*}$. In the former case, $M \not M^{\prime}$ and negative transitivity would give us $M \not M^{*}$, contradicting (ii). In the latter case, $M \not M^{\prime}$ would equally well yield $M \not M^{*}$, again contradicting (ii).

THEOREM 7. Let - be a contraction function over a logically finite theory $A$. Then the following conditions are equivalent:

(i) - is a simple base contraction function;

(ii) - is a prioritized base contraction function;

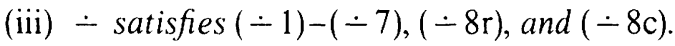

Proof. That (i) entails (ii) is trivial.

To show that (ii) entails (iii), observe that by Theorem $6, \mathscr{C}\left(\left\langle B_{i}\right\rangle\right)=\mathscr{C}\left(\mathscr{S}\left(\mathscr{P}\left(\left\langle B_{i}\right\rangle\right)\right)\right)$ and that $\mathscr{P}\left(\left\langle B_{i}\right\rangle\right)$ is reflexive and negatively transitive. So by Corollary $2, \mathscr{C}\left(\left\langle B_{i}\right\rangle\right)$ satisfies $(-1)-(-7),(-8 \mathrm{r})$, and $(\div 8 \mathrm{c})$.

To show that (iii) entails (i), we conclude from (iii) with the help of Corollary 2 that there is a negatively transitive preference relation $\leq$ such that $-=\mathscr{C}(\mathscr{S}(\leq))$. We know from Lemma 10 that the latter is identical with $\mathscr{C}(\mathscr{S}(\mathscr{P}(\mathscr{B}(\leq))))$. So there 
is a simple belief base $B$, namely $B=\mathscr{B}(\leq)$, such that $\dot{-}=\mathscr{C}(\mathscr{P}(\mathscr{P}(B)))$. By Theorem 6 then, $-=\mathscr{C}(B)$.

Theorem 7 tells us that for every prioritized belief base $\left\langle B_{i}\right\rangle$ for $A$ there is a simple belief base $B$ for $A$ such that $\mathscr{C}(B)=\mathscr{C}\left(\left\langle B_{i}\right\rangle\right)$. In the finite case, every prioritization can be encoded syntactically. Does this mean that prioritization is superfluous? In answering this question we first have to emphasize that our generation of $B$ from $\left\langle B_{i}\right\rangle$ took a rather roundabout route: $B=\mathscr{B}\left(\mathscr{P}\left(\left\langle B_{i}\right\rangle\right)\right)$. An interesting problem now is whether a more perspicuous construction of $B$ from $\left\langle B_{i}\right\rangle$ is possible. This question, too, is put in informal terms, and as such it permits no absolutely precise answer. Still we think that the answer must be no. Even for the most straightforward prioritized belief bases $\left\langle B_{i}\right\rangle$ the generated simple base $B=\mathscr{B}\left(\mathscr{P}\left(\left\langle B_{i}\right\rangle\right)\right)$ becomes grossly unintuitive, and there is no prospect of finding different solutions to the problem. Consider for example the base containing $p$ and $p \rightarrow q$ with the alternative prioritizations $\langle\{p \rightarrow q\},\{p\}\rangle$ and $\langle\{p\},\{p \rightarrow q\}\rangle$. In the former case, $B=\{p \wedge q, p, p \vee q, q \rightarrow p\}$, while in the latter case, $B^{\prime}=\{q, p \leftrightarrow q\}$ will lead to exactly the same results as the prioritized belief base. But in neither case is there anything like a transparent relation to the original base $\left\langle B_{i}\right\rangle$. It appears that prioritization is useful, notwithstanding its formal dispensability in the finite case.

Appendix: Relating theory change and nonmonotonic logic. In a recent paper, Makinson and Gärdenfors [17] make considerable progress toward linking the areas of theory change and nonmonotonic reasoning. They close with the following problem which we quote in full:

We end with an important open question. In their [1], Alchourrón, Gärdenfors, and Makinson established a representation theorem for theory revision operations * satisfying conditions $(* 1)-(* 8)$, in terms of "transitively relational partial meet revisions". The proof went via a representation theorem for a contraction function - satisfying certain conditions $(\div 1)-(-8)$. On the other hand, Kraus, Lehmann, and Magidor [12] have established a representation theorem for supraclassical, cumulative and distributive nonmonotonic inference relations $\sim$ defined between individual propositions, in terms of classical stoppered preferential model structures. The former proof is relatively short and abstract; the latter seems more complex. Also, the latter has not been generalized to a representation theorem for supraclassical, cumulative and distributive inference operations $C: 2^{L} \rightarrow 2^{L}[\ldots]$ Does the representation theorem for theory change hold the key for a solution to this problem of extending the Kraus/Lehmann/Magidor representation theorem to the infinite casedespite the failure of consistency preservation for preferential model structures? Or do we have two essentially different representation problems? ([17, pp. 203-204], notation adapted)

This question does not have a simple answer. Three different points have to be taken into consideration.

First, the approaches of AGM and KLM are not as distinct as Makinson and Gärdenfors seemed to assume. AGM contract and revise by single propositions, 
and similarly KLM consider the nonmonotonic consequences of simple propositions. Makinson and Gärdenfors's equation $y \in A * x$ iff $x \sim y$ (iff $y \in C(x)$ ) fully reflects this. A truly infinitistic stance toward both theory change and nonmonotonic logic is taken only by Lindström [16]. The question of whether the theory $A$ is logically finite has no bearing on this issue. Here Makinson and Gärdenfors saw a difference which simply does not exist.

Secondly, Makinson and Gärdenfors tacitly passed over the fact that in KLM there is no counterpart to $(* 8)$ or $(-8)$. But this difference is indeed crucial, as is clear from a later paper of Lehmann and Magidor [13]. As regards the preferential logics dealt with by KLM, no "relatively short and abstract" proof seems to be possible. Thus, it appears reasonable to construe Makinson and Gärdenfors's question as referring to the rational logics treated in [13].

But thirdly, Lehmann and Magidor's rational logics still differ from AGM-style theory revisions in that they are not required to satisfy a condition of consistency preservation which corresponds to postulate $(-4)$ for contractions. In this respect, Makinson and Gärdenfors show a keen sense of the intricacies of the situation. In unpublished notes, we have applied the techniques developed in this paper to the problem of providing rational logics with canonical models. We have found that it is possible to transfer our proof to the area of nonmonotonic logic, but that consistency preservation with respect to the underlying monotonic logic $C n$ is, in fact, indispensable for a perfect matching. The reason is that the compactness property presupposed for $C n$ runs idle if there are any "inaccessible worlds" (and this is just what a violation of consistency preservation amounts to). Since the results of our efforts bear considerable similarity with the venerable presentation in Lewis [14] except for the fact that the role of Lewis's extra-linguistic propositions (sets of "real" possible worlds) is played by the propositions of the object language - we had better refrain from expounding them here in more detail.

Acknowledgment. I gratefully acknowledge numerous comments and suggestions by David Makinson which have once again been extremcly helpful.

\section{REFFRENCES}

[1] Carlos Alchourrón, Peter Gärieniors, and David Makinson, On the logic of theory change: Partial meet contraction functions and their associated retision functions, this JoURNAL, vol. 50 (1985). pp. $510-530$.

[2] Carlos Alchourrón and David MAKıNSON, Maps hetween some different kinds of contraction function: The finite case, Studia Logica. vol. 45 (1986), pp. 187-198.

[3] Herman ChER NOFf, Rational selection of decision functions, Econometrica, vol. 22(1954). pp. 422443.

[4] André Fuhrmanin and Michael Morreau (editors), The logic of theory change, Leclure Notes in Computer Science, vol. 465, Springer-Verlag, Berlin, 1991.

[5] PETER GÄRDENFORS. Knowledge in flux: Modeling the dynamics of epistemic states, Bradford Books, MIT Press. Cambridge, Massachusetts, 1988.

[6] - (editor). Belief revision. Cambridge University Press, Cambridge. 1992.

[7] Peter Gärdenfors and David Makinson. Revisions of knowledge systems using epistemic entrenchment. Theoretical Aspects of Reasoning About Knowledge (M. Vardi, editor). Morgan Kaufmann, Los Altos, 1988. pp. 83-95. 
[8] Adam Grove, Two modellings for theory change, Journal of Philosophical Logic, vol. 17 (1988). pp. $157-170$.

[9] Sven Ove Hansson, Similarity semantics and minimal changes of helief, Erkenntnis, vol. 37 (1992). pp. $401-429$.

[10] Hans G. Herzberger, Ordinal preference and rational choice. Econometrica, vol. 41 (1973), pp. $187-237$.

[11] Hikofumi Katsuno and Alberto O. Mendelzon, Propositionai knowledge hase retisum and minimal change, Artificial Intelligence, vol. 52 (1991), pp. 263-294.

[12] Sarit Kraus. Daniel Lehmanin, and Menachem Magidor, Nonmonotonic reasoning, preferential models and cumulative logics, Artificial Intelligence, vol. 44 (1990). pp. 167-207.

[13] Daniel Lehmann and MeNachem Magidor, What does a conditional knowledge hase entail:" Artificial Intelligence, vol. 55 (1992), pp. 1-60.

[14] David Lewis, Counterfactuals, Blackwell, Oxford, 1973 (second edition 1986).

[15] - Ordering semantics and premise semantics for counterfactuals, Journal of Philosophical Logic, vol. 10 (1981), pp. 217-234.

[16] StEn Lindström, A semantic approach to nonmonotonic reasoning: Inference and choice. University of Uppsala, April 1991, manuscript.

[17] David Makinson and Peter Gärdenfors, Relations hetween the logic of theory change and nonmonotonic logic, The logic of theory change (A. Fuhrmann and M. Morreau, editors), Lecture Notes in Computer Science, vol. 465, Springer-Verlag, Berlin and New York, 1991, pp. 185-205.

[18] Bernhard Nebel. A knowledge level analysis of helief revision, Proceedings of the Ist international conference on principles of knowledge representation and reasoning ( $R$. Brachman, $H$. Levesque, and R. Reiter, editors), Morgan Kaufmann, San Mateo, California, 1989, pp. 301-311.

[19] - Syntax-hased approaches to belief revision, Belief revision (P. Gärdenfors, editor), Cambridge University Press, Cambridge, 1992, pp. $52-88$.

[20] HaNS RoTT, Two methods of constructing contractions and revisions of knowledge systems. Journal of Philosophical Logic, vol. 20 (1991), pp. 149-173.

[21] - On the logic of theory change: More maps between different kinds of contraction function, Belief revision (P. Gärdenfors, editor), Cambridge University Press, Cambridge, 1992, pp. 122-141.

[22] - Preferential helief change using generalized epistemic entrenchment, Journal of Logic, Language and Information. vol. I (1992), pp. 45-78.

[23] Amartya K. Sen, Choice, welfare, and measurement, Blackwell, Oxford, 1982.

[24] Kotaro Suzumura, Rational choice, collective decisions, and social welfare, Cambridge University Press, Cambridge, 1983.

\author{
FACHGRUPPE PHILOSOPHIE \\ UNIVERSITÄT KONSTANZ. \\ POSTFACH 5560, D-784.34 KONSTAN7. GERMANY
}

E-mail: pirottânyx.uni-konstanz.de 